\title{
ON CONFORMAL MAPS OF INFINITELY CONNECTED DIRICHLET REGIONS $\left(^{1}\right)$
}

\author{
BY \\ V. C. WILLIAMS
}

\begin{abstract}
Let $D$ be a plane region of arbitrary connectivity $(>1)$ for which the Dirichlet problem is solvable. There exists a conformal map of $D$ onto a region bounded by two level loci of $H$, a nontrivial harmonic measure. $H$ is essentially the difference of two logarithmic potentials. The two measures involved are mutually singular probability measures. Further properties of these measures, and of $H$, are derived.

The special case in which $D$ is of connectivity 2 is the classical theorem which states that an annular region is conformally equivalent to a region bounded by two circles. The case in which $D$ is of finite connectivity was treated by J. L. Walsh in 1956.

A similar generalization of the Riemann mapping theorem is also established.

Finally, converses of the above results are also valid.
\end{abstract}

In 1956, J. L. Walsh showed that a plane region bounded by finitely many, mutually disjoint Jordan curves could be mapped conformally onto a region bounded by two level loci of a function harmonic in the plane with the exception of a finite number of poles, one in each component of the complement of the region [11]. This is presented below as Theorem 1 , where $\tau \log T(Z)$ is harmonic, and the region $\Delta$ is defined by $0<\tau \log T(Z)<1$. A special case of Theorem 1 is the classical theorem which states that a doubly connected region can be mapped conformally onto an annulus. Furthermore, Theorem 3 below, due also to J. L. Walsh, is a similar generalization of the Riemann mapping theorem. At least three other proofs of these theorems have since been given (H. Grunsky, [4]; J. A. Jenkins, [7]; H. J. Landau, [8]).

It is the object of this paper to show in Theorems 2 and 4 below that the theorems of Walsh extend further to Dirichlet regions of arbitrary connectivity. Finally, Theorem 5 below can be regarded as a converse of Theorem 2. The existence of the conformal map in Theorem 2 characterizes the Dirichlet region, and thus may prove useful in the study of such regions. Here, a Dirichlet region is one for which the Dirichlet problem is solvable. See [3, p. 53] for a totally unrelated definition of

Presented in part to the Society, January 27, 1963, under the title On conformal maps of regions of infinite connectivity; received by the editors November 20, 1969.

AMS 1969 subject classifications. Primary 3040; Secondary 3075, 3110, 3115.

Key words and phrases. Infinitely connected Dirichlet region, harmonic measure, logarithmic potential, subharmonic function.

(1) This research was supported in part by the U.S. Air Force, Office of Scientific Research of the Air Research and Development Command.

Copyright (C) 1971, American Mathematical Society 
Dirichlet region. A generalization of [11] to finitely connected regions whose boundary components consist of not necessarily disjoint Jordan curves is to be found in [12]. In the work to follow, we shall employ some of the methods of [11] and [12].

The author is indebted to Professors L. V. Ahlfors and J. L. Walsh for advice and encouragement during the early phases of this research.

TheOREM 1 (WALSH). Let $D$ be a region in the extended z-plane. Let the boundary of $D$ consist of mutually disjoint Jordan curves $B_{1}, B_{2}, \ldots, B_{m} ; C_{1}, C_{2}, \ldots, C_{n}$, $m n \neq 0$. There exists a conformal map of $D$ onto a region $\Delta$ of the extended $Z$-plane, one-to-one and continuous in the closures of the two regions, where $\Delta$ is defined by

$$
1<T(Z)<e^{1 / \tau}, \quad T(Z)=A \frac{\prod_{i=1}^{m}\left|Z-a_{i}\right|^{M_{i}}}{\prod_{j=1}^{n}\left|Z-b_{j}\right|^{N_{j}}}
$$

with $\tau, A, M_{i}, N_{j}>0$; and $\sum M_{i}=\sum N_{j}=1$. The locus $T(Z)=1$ consists of $m$ mutually disjoint Jordan curves, respective images of the $B_{i}$, which separate $\Delta$ from the $a_{i}$. The locus $T(Z)=e^{1 / \tau}$ consists of $n$ mutually disjoint Jordan curves, respective images of the $C_{j}$, which separate $\Delta$ from the $b_{j}$.

In Theorem 2 below, the connectivity of the region $D$ is assumed to be at least 2 . If distinct boundary components $B_{1}, B_{2}, \ldots, B_{m} ; C_{1}, C_{2}, \ldots, C_{n}$ of $D$ are specified, it can be shown that the boundary is the union of two disjoint, closed sets $B$ and $C$ such that $B_{i} \subseteq B$ and $C_{j} \subseteq C$.

Theorem 2. Let $D$ be a Dirichlet region in the extended z-plane. Suppose that the boundary points of $D$ are separated into two disjoint, nonempty, closed sets $B$ and $C$. There exists a conformal map of $D$ onto a region $\Delta$ of the extended Z-plane, where $\Delta$ is defined by

$$
1<T(Z)<e^{1 / \tau}, \quad T(Z)=A \frac{\prod_{i}\left|Z-a_{i}\right|^{M_{i}} \exp \int \log |Z-\alpha| d \mu(\alpha)}{\prod_{j}\left|Z-b_{j}\right|^{N_{j}} \exp \int \log |Z-\alpha| d \nu(\alpha)},
$$

with $\tau, A, M_{i}, N_{j}>0$; and $\sum M_{i}, \sum N_{j} \leqq 1$. The measures $\mu$ and $\nu$ are regular Borel measures such that (1) $\mu(\Delta)=\nu(\Delta)=0$, (2) $\mu(Z$-plane $)=1-\sum M_{i} ; \nu(Z$-plane $)=$ $1-\sum N_{j}$, (3) the $\mu$ - and $\nu$-measures of each component of the complement $\Delta^{\prime}$ of $\Delta$ are zero, (4) the $\mu$-and $\nu$-measures of the exterior of $\Delta$ are zero. The function $T(Z)$ is continuous in the closure $\bar{\Delta}$ of $\Delta$ with the values $1, e^{1 / \tau}$ on the boundary of $\Delta$. Furthermore, $T(Z)$ is continuous on each component of $\Delta^{\prime}$ (except at the $b_{j}$ ). The locus $T(Z)=1$ corresponds to the point set $B$ and separates the points $a_{i}$ from each other and from $\Delta$. The locus $T(Z)=e^{1 / \tau}$ corresponds to $C$ and separates the $b_{j}$ from each other and from $\Delta$.

Proof. The proof is long, and consists of 22 propositions. The method is to define an increasing sequence $\left\{D_{k}\right\}$ of subregions of $D$, with $\lim _{k \rightarrow \infty} D_{k}=D$, and such that each $D_{k}$ is bounded by finitely many, mutually disjoint Jordan curves. 
Then Theorem 1 can be applied to each region $D_{k}$. The major part of the proof consists of examining limits.

We assume that both sets $B$ and $C$ consist of infinitely many components. Simple modifications in the proof can be made if this is not the case. Since $B$ and $C$ are closed, there is in $D$ an analytic Jordan curve $\gamma$ which separates $B$ and $C$. The existence of $\gamma$ follows readily from Theorems 17 and 133 in [10, pp. 172, 267]. We may suppose that $0,1, \infty$ are in $D$, and that $B$ is interior to $\gamma$, since these properties may be obtained by a linear transformation of the extended $z$-plane.

Let $u$ be the unique function which is harmonic in $D-\infty$ and continuous in $\bar{D}$, with finite limit at $\infty$, and which takes the value 0 on $B$ and 1 on $C$. For $z$ in $D$, $0<u(z)<1$.

Let $\left\{\varepsilon_{k}\right\}$ be a strictly decreasing sequence of positive numbers with limit 0 . Let

$$
\varepsilon_{1}<\min \{u(z) \mid z=0,1, \infty ; z \in \gamma\}
$$

and

$$
1-\varepsilon_{1}>\max \{u(z) \mid z=0,1, \infty ; z \in \gamma\} .
$$

Since $u$ has at most countably many critical points, we may assume that for all integers $k$, no critical point of $u$ is on any of the loci $u(z)=\varepsilon_{k}$ or $u(z)=1-\varepsilon_{k}$. For each $k$, the locus $u(z)=\varepsilon_{k}$ consists of finitely many, mutually exterior, analytic Jordan curves in $D$ and interior to the curve $\gamma$. Similarly, the locus $u(z)=1-\varepsilon_{k}$ consists of finitely many, mutually exterior, analytic Jordan curves in $D$ but exterior to $\gamma$.

Let $D_{k}=\left\{z \mid \varepsilon_{k}<u(z)<1-\varepsilon_{k}\right\}$. Each $D_{k}$ is a finitely connected subregion of $D$ which contains the points $0,1, \infty$ and the curve $\gamma$. The boundary of $D_{k}$ consists of the loci $u(z)=\varepsilon_{k}$ and $u(z)=1-\varepsilon_{k}$. Since $\varepsilon_{k} \downarrow 0$, and $1-\varepsilon_{k} \uparrow 1$ as $k \rightarrow \infty$, it follows that $D_{1} \subseteq D_{k} \subset D_{k+1} \subset \ldots \subset D$, and $\lim _{k \rightarrow \infty} D_{k}=D$.

We shall have much use for the following lemma:

LemMA 1. Let $E$ be a boundary component of the region $D$. Assume that $\infty \in D$. For each $\varepsilon>0$, there is in $D$ an analytic Jordan curve $\beta$ which encloses $E$ and which is such that every point of the annular region bounded by $\beta$ and $E$ is at a distance less than $\varepsilon$ from some point of $E$.

The lemma is similar to [9, Theorem 1]. It also follows easily from [10, Theorem 17, p. 172].

As a consequence of the lemma, there is in $D$ a sequence $\left\{\beta_{k}\right\}$ of analytic Jordan curves having these properties:

(1) Every $\beta_{k}$ encloses the boundary component $E$.

(2) The $\beta_{k}$ are nested-for each $k, \beta_{k}$ encloses $\beta_{k+1}$.

(3) For each $\varepsilon>0$, there is an integer $K$ such that for $k \geqq K$, every point in the annular region bounded by $\beta_{k}$ and $E$ is at a distance less than $\varepsilon$ from some point of $E$. 
We say that the sequence $\left\{\beta_{k}\right\}$ contracts to $E$.

Suppose now that $E \subset B$. Let $\left\{\beta_{k}\right\}$ be a sequence of analytic Jordan curves in $D$ and interior to $\gamma$, and which contracts to $E$. It is possible to show that the sequence of line integrals

$$
\left\{\int_{\beta_{k}} \frac{\partial u}{\partial n} d s\right\}
$$

is positive and decreasing. Here, and henceforth, $n$ is the exterior normal; $s$ is arc length. Where the line integral is with respect to arc length, as above, the sense of the curve does not matter. It follows that

$$
\lim _{k \rightarrow \infty} \int_{\beta_{k}} \frac{\partial u}{\partial n} d s=\int_{E} \frac{\partial u}{\partial n} d s
$$

exists and is nonnegative. The limit is independent of the sequence $\left\{\beta_{k}\right\}$; another sequence of curves that contracts to $E$ yields the same limit. If $E \subset C$, then the nonnegative number $(-1) \int_{E} \partial u / \partial n d s$ is similarly defined. In this manner, each boundary component of $D$ determines a unique nonnegative number. Of course, $\int_{E} \partial u / \partial n d s$ is not an integral.

The next step is to label inductively the boundary components of the regions $D_{k}$ and $D$. Great care is necessary here in order that we may eventually assert that both $\mu$ - and $\nu$-measures of each component of the complement of the region $\Delta$ are zero. Let the components of the locus $u(z)=\varepsilon_{1}$ be designated $B_{i 1}\left(i=1,2, \ldots, \mu_{1}\right)$ in some arbitrary manner. Let $k \geqq 1$. Suppose that for $1 \leqq t \leqq k$, the components of $u(z)=\varepsilon_{t}$ have been named and are $\left\{B_{i t} \mid i=1,2, \ldots, \mu_{t}\right\}$. Then the curves of $u(z)=$ $\varepsilon_{k+1}$ are labeled as follows:

Select a curve $B_{i k}$. It is possible to show that

$$
\sum \int_{F} \frac{\partial u}{\partial n} d s \leqq \int_{B_{i k}} \frac{\partial u}{\partial n} d s,
$$

where the sum is over any finite set of components $F$ of $B$ that are interior to $B_{i k}$. Thus for each $\rho>0$ there are at most finitely many components $F$ of $B$ interior to $B_{i k}$ such that

$$
\int_{F} \frac{\partial u}{\partial n} d s \geqq \rho .
$$

There is at least one component $E$ interior to $B_{i k}$ for which $\int_{E} \partial u / \partial n d s$ is maximal. The component $E$ is separated from $B_{i k}$ by exactly one curve of the locus $u(z)=\varepsilon_{k+1}$. Call this curve $B_{i k+1}$. Then for $i=1,2, \ldots, \mu_{k}$, the curves $B_{i k+1}$ form part of the locus $u(z)=\varepsilon_{k+1}$. Let the remaining curves (if any) of this locus be designated arbitrarily as $B_{i k+1}\left(i=\mu_{k}+1, \ldots, \mu_{k+1}\right)$. Note that $\mu_{k} \leqq \mu_{k+1}$. Since $B$ is assumed to have infinitely many components, $\mu_{k} \uparrow \infty$ as $k \rightarrow \infty$. If a positive integer $i$ is given, then for $k$ sufficiently large, $\mu_{k} \geqq i$. Thus for such $k$, the sequence $\left\{B_{i k}\right\}$ is defined, and contracts to exactly one component of $B$ as $k \rightarrow \infty$. Call this component $B_{i}$. Remaining components (if any) of $B$ need not be labeled. 
The curves $C_{j k}\left(j=1,2, \ldots, \nu_{k}\right)$ corresponding to the locus $u(z)=1-\varepsilon_{k}$ are similarly defined, as well as components $C_{1}, C_{2}, C_{3}, \ldots$ of $C$.

Since each region $D_{k}$ is bounded by finitely many mutually disjoint Jordan curves, by Theorem 1 , for each $D_{k}$, there exist functions $f_{k}$ and $T_{k}$ such that $f_{k}$ maps $D_{k}$ conformally (except at $\infty$ ) onto a region $\Delta_{k}$ of the extended $Z$-plane, where $\Delta_{k}$ is defined by

$$
1<T_{k}(Z)<\exp \left[1 / \tau_{k}\right], \quad T_{k}(Z)=A_{k} \frac{\prod_{i=1}^{\mu_{k}}\left|Z-a_{i k}\right|^{M_{i k}}}{\prod_{j=1}^{\nu_{k}}\left|Z-b_{j k}\right|^{N_{j k}}},
$$

with $\tau_{k}, A_{k}, M_{i k}, N_{j k}>0$; and $\sum M_{i k}=\sum N_{j k}=1$. We may assume that for every $k$, $f_{k}(0)=0, f_{k}(1)=1$, and $f_{k}(\infty)=\infty$, since these properties may be obtained by a linear transformation of the $Z$-plane. The map $f_{k}$ is one-to-one and continuous from the closure $\bar{D}_{k}$ of $D_{k}$ onto $\bar{\Delta}_{k}$. The locus $T_{k}(Z)=1$ consists of $\mu_{k}$ mutually exterior Jordan curves $B_{i k}^{*}$, respective images of the $B_{i k}$, which separate $\Delta_{k}$ from the points $a_{i k}$; the locus $T_{k}(Z)=\exp \left[1 / \tau_{k}\right]$ consists of $\nu_{k}$ mutually exterior Jordan curves $C_{j k}^{*}$, respective images of the $C_{j k}$, which separate $\Delta_{k}$ from the points $b_{j k}$. Also, $\bigcup_{i=1}^{\mu_{k}} B_{i k}^{*}$ is interior to the Jordan curve $f_{k}(\gamma) ; \bigcup_{j=1}^{v_{k}} C_{j k}^{*}$ is exterior to $f_{k}(\gamma)$.

Let $u_{k}(z)=\left[u(z)-\varepsilon_{k}\right] /\left(1-2 \varepsilon_{k}\right), z \in \bar{D}$. Since $\left|u-u_{k}\right| \leqq \varepsilon_{k} /\left(1-2 \varepsilon_{k}\right), \lim _{k \rightarrow \infty} u_{k}(z)$ $=\dot{u}(z)$ uniformly in $\bar{D}$. The function $u_{k}$ is the unique function which is harmonic in $D_{k}-\infty$ and continuous in $\bar{D}_{k}$, with finite limit at $\infty$, and which takes the boundary values 0 on $\bigcup_{i=1}^{\mu_{k}} B_{i k \text {. }}$ and 1 on $\bigcup_{j=1}^{\nu_{k}} C_{j k}$.

Proposition 1. $\lim \tau_{k}=\tau$ exists; $\tau_{k} \downarrow \tau$ as $k \rightarrow \infty ; 0<\tau<\infty$.

Proof. The functions $u_{k}(z)$ and $\tau_{k} \log T_{k}\left[f_{k}(z)\right]$ coincide in $D_{k}$, since each is harmonic and bounded in $D_{k}-\infty$ with boundary values 0 on $\bigcup_{i=1}^{\mu_{k}} B_{i k}$ and 1 on $\bigcup_{j=1}^{v_{k}} C_{j k}$. For each $k$, the points $a_{i k}$ are interior to the Jordan curve $f_{k}(\gamma)$; the points $b_{j k}$ are exterior to $f_{k}(\gamma)$. Thus

$$
\begin{aligned}
\tau_{k} & =\tau_{k} \sum_{i=1}^{\mu_{k}} M_{i k}=\frac{\tau_{k}}{2 \pi} \int_{f_{k}(\gamma)} \frac{\partial}{\partial n} \log T_{k}(Z) d s \\
& =\frac{1}{2 \pi} \int_{\gamma} \frac{\partial}{\partial n} \tau_{k} \log T_{k}\left[f_{k}(z)\right] d s=\frac{1}{2 \pi} \int_{\gamma} \frac{\partial u_{k}}{\partial n} d s \\
& =\frac{1}{2 \pi\left(1-2 \varepsilon_{k}\right)} \int_{\gamma} \frac{\partial u}{\partial n} d s .
\end{aligned}
$$

Then

$$
\lim _{k \rightarrow \infty} \tau_{k}=\frac{1}{2 \pi} \int_{\gamma} \frac{\partial u}{\partial n} d s=\tau
$$

exists. Since $\tau_{k}>0, \int_{\gamma} \partial u / \partial n d s>0$; thus $0<\tau<\infty$. Since $\varepsilon_{k} \downarrow 0$ as $k \rightarrow \infty, \tau_{k} \downarrow \tau$ as $k \rightarrow \infty$.

Proposition 2. $\lim _{k \rightarrow \infty} A_{k}=A$ exists $; 1<A<e^{1 / \tau}$. 
Proof. As in Proposition $1, u_{k}(z)=\tau_{k} \log T_{k}(Z)$, where $Z=f_{k}(z)$. As $z \rightarrow \infty$, $f_{k}(z) \rightarrow \infty$. Thus, taking limits, we obtain $u_{k}(\infty)=\tau_{k} \log T_{k}(\infty)=\tau_{k} \log A_{k}$. Hence $A_{k}=\exp \left[u_{k}(\infty) / \tau_{k}\right]$, and $\lim _{k \rightarrow \infty} A_{k}=\exp [u(\infty) / \tau]=A$ exists. Since $0<u(\infty)<1$, $1<A<e^{1 / \tau}$.

Proposition 3. $\lim _{k \rightarrow \infty} M_{i k}=M_{i}$ and $\lim _{k \rightarrow \infty} N_{j k}=N_{j}$ exist; $M_{i}$ and $N_{j} \geqq 0$; $\sum_{i} M_{i}, \sum_{j} N_{j} \leqq 1$.

Proof. Choose $i$. For $k$ sufficiently large, $i \leqq \mu_{k}$. Then

$$
M_{i k}=\frac{1}{2 \pi \tau_{k}} \int_{B_{i k}} \frac{\partial u_{k}}{\partial n} d s=\frac{1}{2 \pi \tau_{k}\left(1-2 \varepsilon_{k}\right)} \int_{B_{i k}} \frac{\partial u}{\partial n} d s .
$$

Since $\tau_{k} \rightarrow \tau, \varepsilon_{k} \rightarrow 0$, and $B_{i k}$ contracts to $B_{i}$ as $k \rightarrow \infty$,

$$
\lim _{k \rightarrow \infty} M_{i k}=\frac{1}{2 \pi \tau} \int_{B_{i}} \frac{\partial u}{\partial n} d s=M_{i}
$$

exists. Since all $M_{i k}>0, M_{i} \geqq 0$. It is not difficult to show that if $B_{i}$ is an isolated boundary component of $D$ then $M_{i}>0$; and if $B_{i}$ consists of a single point, then $M_{i}=0$.

Similarly,

$$
\lim _{k \rightarrow \infty} N_{j k}=\frac{-1}{2 \pi \tau} \int_{C_{j}} \frac{\partial u}{\partial n} d s=N_{j}
$$

exists and is $\geqq 0$.

Let $t$ be a positive integer. For $k$ sufficiently large, $t \leqq \mu_{k}$. Then $\sum_{i=1}^{t} M_{i k} \leqq$ $\sum_{i=1}^{\mu_{k}} M_{i k}=1$. Letting $k \rightarrow \infty$, obtain $\sum_{i=1}^{t} M_{i} \leqq 1$. Since $t$ is arbitrary, $\sum_{i=1}^{\infty} M_{i} \leqq 1$.

By the same reasoning, $\sum_{j=1}^{\infty} N_{j} \leqq 1$, and Proposition 3 is established.

In $D-\{0,1, \infty\}$, the functions $f_{k}$ are analytic and do not take the values 0,1 and consequently form a normal family. It is easily shown that the family $\left\{f_{k}\right\}$ is actually normal in $D-\infty$. Thus there is a subsequence $\left\{k^{\prime}\right\}$ of the integers and a function $f$ such that $\lim _{k^{\prime} \rightarrow \infty} f_{k^{\prime}}(z)=f(z)$ uniformly on each compact subset of $D-\infty$. Since $f(0)=0, f(1)=1, f$ is not identically constant, and thus is one-to-one and analytic in $D-\infty$. Furthermore, $f$ has a simple pole at $\infty$. If we define $f(\infty)=\infty$, then $Z=f(z)$ determines a continuous univalent map of $D$ onto a region $\Delta$ of the extended $Z$-plane. The map is conformal in $D-\infty$.

Now and hereafter, a rational, half-open rectangle is understood to be one with rational points as vertices, with edges parallel to the coordinate axes, and containing the points of its left and lower edges, but not those of its upper or right edges. There are countably many such rectangles. For $k=1,2,3, \ldots$, and for each rational, half-open rectangle $R$ in the $Z$-plane, let

$$
m_{k}(R)=\sum_{i}\left(M_{i k}-M_{i}\right)
$$


where the sum is over all $i$ for which $a_{i k} \in R$. Similarly, let

$$
n_{k}(R)=\sum_{j}\left(N_{j k}-N_{j}\right)
$$

where the sum is over all $j$ for which $b_{j k} \in R$.

By Cantor's diagonal process, there is a subsequence $\left\{k^{\prime \prime}\right\}$ of the sequence $\left\{k^{\prime}\right\}$ such that for each rational, half-open rectangle $R, \lim _{k^{\prime \prime} \rightarrow \infty} m_{k^{\prime \prime}}(R)=m(R)$ and $\lim _{k^{\prime \prime} \rightarrow \infty} n_{k^{\prime \prime}}(R)=n(R)$ exist, and for each $i, \lim _{k^{\prime \prime} \rightarrow \infty} a_{i k^{\prime \prime}}=a_{i}$ exists, and for each $j$, $\lim _{k^{\prime \prime} \rightarrow \infty} b_{j k^{\prime \prime}}=b_{j}$ exists. Henceforth, we restrict ourselves to a definite subsequence of integers $k$ for which the limits $m(R), n(R), a_{i}, b_{j}$ (finite or infinite) exist and also for which $\lim _{k \rightarrow \infty} f_{k}(z)=f(z)$ uniformly on each compact subset of $D-\infty$.

There are sets $S$ in the extended $Z$-plane other than rational half-open rectangles for which $\lim m_{k}(S)$ exists. In the case of such $S$, define $m(S)$ as the limit of $m_{k}(S)$. The set function $n$ can be similarly extended.

Proposition 4. The sets $\left\{a_{i k}\right\}$ and $\left\{b_{j k}\right\}$ are bounded. Consequently, the sets $\left\{a_{i}\right\}$ and $\left\{b_{j}\right\}$ of limits are also bounded.

Proof. Let $\beta$ be a circle in $D$ that encloses the complement of $D$. Since $f_{k} \rightarrow f$ uniformly on $\beta$, there exists an integer $K$ such that for $k \geqq K, D_{k}$ contains $\beta$, and $\left|f_{k}(z)\right|<1+\max \{|f(w)| \mid w \in \beta\}=P$ for all $z \in \beta$. Since for $k \geqq K$, the points $a_{i k}$ are interior to the Jordan curve $f_{k}(\beta),\left|a_{i k}\right| \leqq P$. The set $\left\{a_{i k} \mid k<K\right\}$ is finite, thus is bounded. Therefore $\left\{a_{i k}\right\}$ is bounded. By the same reasoning, $\left\{b_{j k}\right\}$ is bounded. Proposition 4 is established.

For $i=1,2,3, \ldots$, let $B_{i}^{*}$ be the boundary component of $\Delta$ that corresponds to the boundary component $B_{i}$ of $D$. That is, $z$ in $D$ tends to $B_{i}$ if and only if $f(z)$ in $\Delta$ tends to $B_{i}^{*}$. Equivalently, $\alpha$ is a Jordan curve in $D$ that encloses $B_{i}$ if and only if $f(\alpha)$ is a Jordan curve in $\Delta$ that encloses $B_{i}^{*}$. Similarly, for $j=1,2,3, \ldots$, let $C_{j}^{*}$ correspond to $C_{j}$. We shall have occasion now and later to use the following lemma, a generalization of Hurwitz's theorem [2, $\$ 120$, p. 74].

LEMMA 2. Let $\left\{f_{k}\right\}$ be a sequence of one-to-one analytic functions. Suppose that as $k \rightarrow \infty, f_{k} \rightarrow f$ uniformly on each compact subset of a region $D$. Suppose that $f$ is not identically constant, and thus is one-to-one and analytic. If $Q$ is a compact subset of the range of $f$, then for each $k$ sufficiently large, $Q$ is contained in the range of $f_{k}$.

A proof of the lemma can be found in [11]. The.same proof is reproduced in [8]. Here is a sketch of an indirect proof similar to the one in [2] that makes use of Hurwitz's theorem.

If the lemma is not valid, then by restricting to a subsequence of $\{k\}$, we may obtain a sequence $\left\{Z_{k}\right\}$ in the range of $f$ with limit $Z_{0}$ also in the range of $f$ such that $f_{k}(z) \neq Z_{k}$. Then $\left\{f_{k}(z)-Z_{k}\right\}$ converges uniformly on each compact subset of $D$ to $f(z)-Z_{0}$. But the functions $f_{k}(z)-Z_{k}$ are never zero, whereas $f(z)-Z_{0}$ has exactly one zero in $D$, contrary to Hurwitz's theorem. 
Proposition 5. For each $i, a_{i}$ is on the component of $\Delta^{\prime}$ that is bounded by $B_{i}^{*}$. Similarly, for each $j, b_{j}$ is on the component of $\Delta^{\prime}$ bounded by $C_{j}^{*}$.

Proof. Let $Z_{0} \neq \infty$ be in $\Delta$. For some $r>0$, the disk $\left|Z-Z_{0}\right| \leqq r$ is in $\Delta$. Since $f_{k} \rightarrow f$ uniformly on each compact subset of $D-\infty$, by Lemma 2 , for $k$ sufficiently large the disk is contained in $\Delta_{k}$. Since no $a_{i k}$ or $b_{j k}$ is in $\Delta_{k},\left|a_{i k}-Z_{0}\right|$ and $\left|b_{j k}-Z_{0}\right|$ $>r$; thus, $Z_{0} \neq a_{i}, \neq b_{j}$. Therefore, no $a_{i}$ or $b_{j}$ is in $\Delta$.

Choose $i$. Let $\beta$ be an arbitrary analytic Jordan curve in $\Delta$ which encloses $B_{i}^{*}$. Let $\alpha=f^{-1}(\beta)$; $\alpha$ encloses $B_{i}$. For $k$ sufficiently large, $\alpha$ is in $D_{k}$. For such $k, a_{i k}$ is interior to $f_{k}(\alpha)$. Then, as in $[11$, p. 133],

$$
\frac{1}{2 \pi i} \int_{\alpha} \frac{f_{k}^{\prime}(z) d z}{f_{k}(z)-a_{i k}}=1
$$

Since $a_{i} \in \Delta^{\prime}, f$ does not take the value $a_{i}$ on $\alpha$. Thus, letting $k \rightarrow \infty$, we obtain

$$
\frac{1}{2 \pi i} \int_{\alpha} \frac{f^{\prime}(z) d z}{f(z)-a_{i}}=1 .
$$

Therefore $a_{i}$ is interior to $f(\alpha)=\beta$. As a consequence of Lemma 1, we may conclude that $a_{i}$ is either on $B_{i}^{*}$ or separated from $\Delta$ by $B_{i}^{*}$.

Proposition 6. Let $\left|Z-a_{i}\right|^{M_{i}}=1$ if $M_{i}=0$. The product $\prod_{i=1}^{\infty}\left|Z-a_{i}\right|^{M_{i}}$ converges absolutely or it diverges to zero. Thus it is defined for each $Z \neq \infty$. The product converges uniformly on every bounded, compact subset of the Z-plane disjoint from the closure of $\left\{a_{i}\right\}$.

Analogous statements hold for $\prod_{j=1}^{\infty}\left|Z-b_{j}\right|^{N_{j}}$.

Proof. Choose $Z \neq \infty$. Choose $R>1$ and such that $\left|Z-a_{i}\right| \leqq R$ for all $i$. Let $s$ be any positive integer. Suppose that

$$
\liminf _{k \rightarrow \infty} \prod_{i=s}^{k}\left|Z-a_{i}\right|^{M_{i}}=r .
$$

Obviously $0 \leqq r \leqq R$. Let $\varepsilon>0$ be given. Since $\sum_{1}^{\infty} M_{i}$ converges, there is an integer $K$ such that for $k \geqq K$ and $t \geqq 1$,

$$
\prod_{k+1}^{k+t} R^{M_{i}}<1+\varepsilon /(2 r+\varepsilon) .
$$

Let $k$ be an integer $\geqq K$ such that

$$
\prod_{i=s}^{k}\left|Z-a_{i}\right|^{M_{i}}<r+\varepsilon / 2
$$

Then for $t \geqq 1$,

$$
\begin{aligned}
\prod_{i=s}^{k+t}\left|Z-a_{i}\right|^{M_{i}} & \leqq(r+\varepsilon / 2) \prod_{k+1}^{k+t} R^{M_{i}} \\
& <(r+\varepsilon / 2)[1+\varepsilon /(2 r+\varepsilon)]=r+\varepsilon .
\end{aligned}
$$


Therefore

$$
\limsup _{t \rightarrow \infty} \prod_{i=s}^{k+t}\left|Z-a_{i}\right|^{M_{i}} \leqq r+\varepsilon .
$$

It follows immediately that

$$
\prod_{i=s}^{\infty}\left|Z-a_{i}\right|^{M_{i}}=r .
$$

The limit exists for each $Z \neq \infty$, and for each positive integer $s$.

Suppose that for some $s, \prod_{i=s}^{\infty}\left|Z-a_{i}\right|^{M_{i}}$ is positive. Then $\sum_{i=s}^{\infty} M_{i} \log \left|Z-a_{i}\right|$ converges. If in this series, we replace all negative terms by 0 , the resulting new series is dominated by $\sum_{s}^{\infty} M_{i} \log R$, and thus converges. It follows that the original series must converge absolutely. Therefore, $\prod_{i=s}^{\infty}\left|Z-a_{i}\right|^{M_{i}}$ and also $\prod_{i=1}^{\infty}\left|Z-a_{i}\right|^{M_{i}}$ converge absolutely.

If $\prod_{i=s}^{\infty}\left|Z-a_{i}\right|^{M_{i}}=0$ for all $s$, then $\prod_{i=1}^{\infty}\left|Z-a_{i}\right|^{M_{i}}$ diverges to zero. In this case also the value of the product is independent of the order of the factors.

The proof of the statement about uniform convergence is routine and is omitted.

To obtain a product with positive exponents $M_{i}$, delete those factors having zero exponents.

Proposition 7. Let $S$ be a subset of the Z-plane. For $k=1,2,3, \ldots$, let $m_{k}(S)$ $=\sum_{i}\left(M_{i k}-M_{i}\right)$, summed over all $i$ such that $a_{i k} \in S$. Then $\lim _{\inf _{k \rightarrow \infty}} m_{k}(S) \geqq 0$. Similarly, $\lim \inf n_{k}(S)=\lim \inf \sum_{j}\left(N_{j k}-N_{j}\right) \geqq 0$. Here the sum is over all $j$ for which $b_{j k} \in S$.

Proof. $\lim \inf m_{k}(S)$ exists since $\left|m_{k}(S)\right| \leqq 1$. Let $r$ be a positive integer. We have

$$
m_{k}(S) \geqq \sum_{i}\left(M_{i k}-M_{i}\right)-\sum_{i=r}^{\infty} M_{i},
$$

where the first sum is over all $i<r$ such that $a_{i k} \in S$. Then

$$
m_{k}(S) \geqq-\sum_{i=1}^{r-1}\left|M_{i k}-M_{i}\right|-\sum_{i=r}^{\infty} M_{i} .
$$

Since $M_{i k} \rightarrow M_{i}$ as $k \rightarrow \infty$,

$$
\liminf _{k \rightarrow \infty} m_{k}(S) \geqq-\sum_{i=r}^{\infty} M_{i}
$$

Letting $r \rightarrow \infty$, obtain lim $\inf _{k \rightarrow \infty} m_{k}(S) \geqq 0$.

We observe that the set functions $m_{k}$ and $n_{k}$ are additive, but not necessarily nonnegative, or monotone.

Proposition 8. The set functions $m$ and $n$ are nonnegative, finitely additive, and monotone. 
Proof. Since $m=\lim _{k \rightarrow \infty} m_{k}$, nonnegativeness follows from the preceding proposition.

That $m$ is finitely additive follows easily from the fact that each $m_{k}$ is finitely additive.

Finally, to show that $m$ is monotone, let $S \subseteq S_{0}$, where both $m(S)$ and $m\left(S_{0}\right)$ are defined. Then $m_{k}\left(S_{0}\right)=m_{k}(S)+m_{k}\left(S_{0}-S\right)$. Since the limit as $k \rightarrow \infty$ exists for two of these terms, it also exists for the third, and $m\left(S_{0}\right)=m(S)+m\left(S_{0}-S\right)$. Since $m\left(S_{0}-S\right) \geqq 0, m(S) \leqq m\left(S_{0}\right)$.

The same proof holds for the set function $n$.

Proposition 9. Let $S$ be a subset of the extended Z-plane whose closure is disjoint from that part of $\Delta^{\prime}$ lying interior to the Jordan curve $\Gamma=f(\gamma)$. Then $m(S)$ is defined and is zero. Similar reasoning shows that if $\bar{S}$ is disjoint from the part of $\Delta^{\prime}$ exterior to $\Gamma$, then $n(S)=0$. Thus, if $\bar{S} \subset \Delta$, then $m(S)=n(S)=0$.

Proof. Let $\beta$ be a Jordan curve in $\Delta$ which encloses $\Gamma$ and which is such that the annular region bounded by $\beta$ and $\Gamma$ is contained in $\Delta$. Let $S_{1}$ consist of the points of $S$ on or interior to $\beta$. Since $\bar{S}_{1}$ is a compact subset of $\Delta-\infty$, by Lemma 2, there is an integer $K_{1}$ such that for $k \geqq K_{1}, \bar{S}_{1} \subseteq \Delta_{k}$. Thus for $k \geqq K_{1}, a_{i k} \notin S_{1}$.

Since $f_{k} \rightarrow f$ uniformly on $\gamma$, there is an integer $K_{2}$ such that for $k \geqq K_{2}, f_{k}(\gamma)$ is a Jordan curve lying interior to $\beta$. Since for fixed $k$, the $a_{i k}$ are interior to $f_{k}(\gamma)$, such $a_{i k}$ are interior to $\beta$ and thus are not in $S-S_{1}$. It follows that for $k \geqq \max \left\{K_{1}, K_{2}\right\}$, $a_{i k} \notin S$; thus $m_{k}(S)=0$. Therefore $m(S)=\lim _{k \rightarrow \infty} m_{k}(S)=0$.

One fact of interest is that $m(\infty)=0$. Furthermore, if $m(S)$ is defined, then $m(S-\infty)$ is also defined, and $m(S)=m(S-\infty)$.

A similar proof establishes the analogous statements about $n$. However, a slight modification is necessary if $S$ is unbounded. In this case it is convenient to use the fact that $\left\{b_{j k}\right\}$ is a bounded set. Details are omitted.

Proposition 10. $m(Z$-plane $)=1-\sum_{i=1}^{\infty} M_{i}$. Similarly, $n(Z$-plane $)=1-\sum_{j=1}^{\infty} N_{j}$.

Proof. $m_{k}(Z$-plane $)=\sum_{i=1}^{\mu_{k}}\left(M_{i k}-M_{i}\right)=1-\sum_{i=1}^{\mu_{k}} M_{i}$. Letting $k \rightarrow \infty$, we obtain $m(Z$-plane $)=1-\sum_{i=1}^{\infty} M_{i}$.

We observe that if $m(S)$ is defined, then $m\left(S^{\prime}\right)$ is also defined, and $m(S)+m\left(S^{\prime}\right)$ $=m(Z$-plane $)$.

Let $R$ be any rational, half-open rectangle whose interior contains $\Delta^{\prime}$. As a consequence of Propositions 9 and $10, m(R)=1-\sum_{i=1}^{\infty} M_{i}$, and $m\left(R^{\prime}\right)=0$. Let $\phi$ be a real-valued continuous function defined on the complex plane. Let $P$ be a subdivision of $R$ into finitely many, mutually disjoint, rational, half-open rectangles $r_{1}, r_{2}, \ldots, r_{t}$. Let norm $P$ be the maximum of the diameters of the $r_{i}$. Choose $\alpha_{i} \in r_{i}$. Then, just as in elementary integration theory,

$$
\lim _{\operatorname{norm} P \rightarrow 0} \sum_{i=1}^{t} \phi\left(\alpha_{i}\right) m\left(r_{i}\right)=M(\phi)
$$


exists. $M$ is a linear functional on the real-valued continuous functions of a complex variable. The functional $M$ is independent of the choice of the rectangle $R$. All we require is that $m(R)=1-\sum_{i=1}^{\infty} M_{i}$. The function $\phi$ may be redefined exterior to $R$ so as to be 0 in a neighborhood of $\infty$, and $M(\phi)$ is unchanged. In fact $\phi$ need not even be defined except in $\bar{R}$. Since the set function $m$ is nonnegative, $M$ is nonnegative. By Riesz's representation theorem [5, Theorem E, p. 234; Theorem D, p. 247], there exists a regular Borel measure $\mu$ such that $M(\phi)=\int \phi d \mu$ for every real-valued function $\phi$ continuous in the complex plane and vanishing in a neighborhood of $\infty$.

Similarly, there is a linear functional $N$ that can be defined using the set function $n$, and a regular Borel measure $\nu$ such that $N(\phi)=\int \phi d \nu$.

Proposition 11. Let $S$ be a nonempty open subset of the Z-plane. Then $\mu(S)$ $\leqq \lim \inf _{k \rightarrow \infty} m_{k}(S)$. Similarly, $\nu(S) \leqq \lim \inf _{k \rightarrow \infty} n_{k}(S)$.

Proof. Let $\left\{S_{t}\right\}$ be a sequence of bounded open subsets of $S$ such that for $t=1,2,3, \ldots, \bar{S}_{t} \subset S_{t+1}$, and $\lim _{t \rightarrow \infty} S_{t}=S$. Let continuous functions $g_{t}$ be defined as follows:

(1) $g_{t}=1$ on $\bar{S}_{t}$;

(2) $g_{t}=0$ on $S_{t+1}^{\prime}$;

(3) $0<g_{t}<1$ on $S_{t+1}-\bar{S}_{t}$.

Then

$$
\mu\left(S_{\iota}\right) \leqq \int g_{t} d \mu=M\left(g_{t}\right)
$$

For each $t, S_{t+1}$ can be covered by finitely many, mutually disjoint, rational, halfopen rectangles $s_{1}, s_{2}, \ldots, s_{r}$ whose union $S_{0}$ is in $S$. Since $g_{t} \leqq 1$ in $S_{0}$, and $g_{t}=0$ in $S_{0}^{\prime}$, it is clear that $M\left(g_{t}\right) \leqq \sum_{i=1}^{r} m\left(s_{i}\right)=m\left(S_{0}\right)$. Since $m_{k}\left(S_{0}\right)+m_{k}\left(S-S_{0}\right)=m_{k}(S)$, we have

$$
m\left(S_{0}\right)+\liminf _{k \rightarrow \infty} m_{k}\left(S-S_{0}\right)=\liminf _{k \rightarrow \infty} m_{k}(S)
$$

Then, as a consequence of Proposition 7, $m\left(S_{0}\right) \leqq \lim \inf _{k \rightarrow \infty} m_{k}(S)$. It follows that $\mu\left(S_{t}\right) \leqq \lim \inf _{k \rightarrow \infty} m_{k}(S)$. Letting $t \rightarrow \infty$, obtain $\mu(S) \leqq \lim _{\inf } \rightarrow \infty m_{k}(S)$.

Proposition 12. If $\phi$ is a real-valued continuous function defined in the complex plane, then $M(\phi)=\int \phi d \mu ; N(\phi)=\int \phi d \nu$.

Proof. Let $d$ be an open disk whose closure is in $\Delta-\infty$. By Proposition 9, $m(d)=0$. By Proposition $11, \mu(d) \leqq m(d)$; thus $\mu(d)=0$. Since $\Delta-\infty$ is a countable union of such disks, $\mu(\Delta-\infty)=0$.

Let $R$ be a rational half-open rectangle whose interior contains $\Delta^{\prime}$. Let $\phi_{1}$ be a real-valued continuous function which coincides with $\phi$ in $R$, but which vanishes in a neighborhood of $\infty$. Since $\phi_{1}=\phi$ except perhaps on a subset of $\Delta-\infty$,

$$
M(\phi)=M\left(\phi_{1}\right)=\int \phi_{1} d \mu=\int \phi d \mu .
$$


The same reasoning shows that $N(\phi)=\int \phi d \nu$.

Proposition 13. $\mu(Z$-plane $)=1-\sum_{i=1}^{\infty} M_{i}$. Similarly, $\nu(Z$-plane $)=1-\sum_{j=1}^{\infty} N_{j}$.

Proof. $\mu(Z$-plane $)=\int 1 d \mu=M(1)=1-\sum_{i=1}^{\infty} M_{i}$.

Let $S$ be a subset of the extended $Z$-plane. Then $S$ is a Borel subset of the extended $Z$-plane if and only if $S-\infty$ is a Borel subset of the (finite) $Z$-plane. Thus we may extend the measures $\mu$ and $\nu$ to measures on the Borel subsets of the extended $Z$-plane by the equations

$$
\mu(S)=\mu(S-\infty), \quad \nu(S)=\nu(S-\infty),
$$

for every Borel subset $S$ in the extended Z-plane. Since $\mu(\infty)=\nu(\infty)=0$, the $\mu$ and $\nu$-measures of the extended plane are $1-\sum M_{i}$ and $1-\sum N_{j}$ respectively. Thus these extended measures are still Borel measures.

As a consequence of the proof of Proposition 12, $\mu(\Delta)=\nu(\Delta)=0$. Thus $\mu\left(\Delta^{\prime}\right)$ $=1-\sum M_{i}$ and $\nu\left(\Delta^{\prime}\right)=1-\sum N_{j}$.

Proposition 14. Let $E$ be a component of the boundary of $D$. If $E \neq B_{i}, E \neq C_{j}$, then $\int_{E} \partial u / \partial n d s=0$.

Proof. Suppose $E$ is a component of $B$, and that

$$
\int_{E} \frac{\partial u}{\partial n} d s=\varepsilon>0
$$

Let $\beta$ be an analytic Jordan curve in $D$, interior to $\gamma$, enclosing $E$, and such that

$$
\varepsilon \leqq \int_{\beta} \frac{\partial u}{\partial n} d s<2 \varepsilon
$$

Let $k$ be such that $\varepsilon_{k}$ is less than the minimum of $u$ on $\beta$. Then that part of the locus $u(z)=\varepsilon_{k}$ that lies interior to $\beta$ consists of one or more analytic Jordan curves. For some $i \leqq \mu_{k}, B_{i k}$ is the curve that separates $E$ from $\beta$. Suppose $F$ is another component of $B$ interior to $B_{i k}$. Since

$$
\int_{E} \frac{\partial u}{\partial n} d s+\int_{F} \frac{\partial u}{\partial n} d s \leqq \int_{\beta} \frac{\partial u}{\partial n} d s<2 \varepsilon
$$

we have

$$
\int_{F} \frac{\partial u}{\partial n} d s<\varepsilon=\int_{E} \frac{\partial u}{\partial n} d s
$$

Evidently, $E$ is the component of $B$ interior to $B_{i k}$ for which the number $\int_{E} \partial u / \partial n d s$ is maximal. Hence the curve $B_{i k+1}$ also encloses $E$; and thus $E=B_{i}$.

Similarly, if $E$ is a component of $C$ with

$$
(-1) \int_{E} \frac{\partial u}{\partial n} d s>0
$$

then for some $j, E=C_{j}$. 
Proposition 15. For every component $Q$ of $\Delta^{\prime}, \mu(Q)=\nu(Q)=0$.

Proof. Suppose first that $Q$ is exterior to $\Gamma=f(\gamma)$. Let $S$ be the unbounded region determined by $\Gamma$. By Proposition $9, m(S)=0$. Then, as a consequence of Proposition 11 , we have $\mu(S)=0$. Since $Q \subset S, \mu(Q)=0$.

Assume $Q$ is interior to $\Gamma$. Then $Q$ corresponds to a component of $D^{\prime}$ lying in the $z$-plane and interior to the curve $\gamma$. Let the boundary of this component be $E$, which is also one of the components of the boundary of $D$.

Let $\varepsilon>0$ be given. Suppose that for some $t, E=B_{t}$. Let $\beta$ be an analytic Jordan curve in $D$ and interior to $\gamma$, enclosing $E$ and for which

$$
\frac{1}{2 \pi \tau} \int_{\beta} \frac{\partial u}{\partial n} d s-M_{t}<\varepsilon
$$

In case $E \neq B_{t}$ for any $t$, by Proposition 14 we can choose $\beta$ so that

$$
\frac{1}{2 \pi \tau} \int_{B} \frac{\partial u}{\partial n} d s<\varepsilon .
$$

Let $\eta=f(\beta) ; \eta$ is interior to $\Gamma$ and encloses $Q$. Let $S$ be the region interior to $\eta$. Let $\zeta$ be a Jordan curve in $\Delta$ and interior to $\eta$ which encloses $Q$ and which is such that the annular region bounded by $\zeta$ and $\eta$ is in $\Delta$. There is an integer $K$ such that for each $k \geqq K, \beta \subset D_{k}, \Delta_{k}$ covers the closed region bounded by $\zeta$ and $\eta$, and the Jordan curve $\eta_{k}=f_{k}(\beta)$ encloses $\zeta$.

For $k \geqq K$, if $a_{i k}$ is interior to $\eta$, then $a_{i k}$ is interior to $\eta_{k}$. If $E=B_{t}$, then $a_{t} \in Q$. In this case, since $a_{t k} \rightarrow a_{t}$, we may assume also that $a_{t k} \in S$ for $k \geqq K$. Then

$$
\begin{aligned}
m_{k}(S) & =\sum\left(M_{i k}-M_{i}\right) \leqq\left(\sum M_{i k}\right)-M_{t} \\
& =\frac{1}{2 \pi} \int_{\eta_{k}} \frac{\partial}{\partial n} \log T_{k}(Z) d s-M_{t} \\
& =\frac{1}{2 \pi \tau_{k}} \int_{\beta} \frac{\partial u_{k}}{\partial n} d s-M_{t},
\end{aligned}
$$

where the first sum above is over all integers $i$ such that $a_{i k} \in S$, but the second is over all $i$ such that $a_{i k}$ is interior to $\eta_{k}$. Letting $k \rightarrow \infty$, we obtain

$$
\liminf _{k \rightarrow \infty} m_{k}(S) \leqq \frac{1}{2 \pi \tau} \int_{\beta} \frac{\partial u}{\partial n} d s-M_{t}<\varepsilon .
$$

If $E \neq B_{t}$, delete $M_{t}$ above. In either case, as a consequence of Proposition 11, we have $\mu(S)<\varepsilon$. Since $Q \subset S, \mu(Q)<\varepsilon$; thus $\mu(Q)=0$.

By a similar argument, $\nu(Q)=0$.

From this proposition and the fact that an open set is a countable union of open components, it follows readily that the $\mu$ - and $\nu$-measures of the exterior of $\Delta$ are zero. 
Proposition 16. Let

$$
T(Z)=A \frac{\prod_{i=1}^{\infty}\left|Z-a_{i}\right|^{M_{i}} \exp \int \log |Z-\alpha| d \mu(\alpha)}{\prod_{j=1}^{\infty}\left|Z-b_{j}\right|^{N_{j}} \exp \int \log |Z-\alpha| d \nu(\alpha)}
$$

Let $\Delta^{1}$ be a compact subset of $\Delta-\infty$. Then $\lim _{k \rightarrow \infty} \log T_{k}(Z)=\log T(Z)$ uniformly on $\Delta^{1}$.

Proof. First, we shall show that the sequence $\left\{\log T_{k}\right\}$ has the uniform Cauchy property on $\Delta^{1}$, and thus converges uniformly on $\Delta^{1}$. Then we shall show that $\log T_{k}(Z) \rightarrow \log T(Z)$ pointwise in $\Delta-\infty$.

It is sufficient to consider only the case that $\Delta^{1}$ is a closed disk of positive radius $r$ and such that the concentric closed disks $\Delta^{2}$ and $\Delta^{3}$ of radii $2 r$ and $3 r$ respectively are also in $\Delta$. By Lemma 2 , there is an integer $K_{1}$ such that $\Delta^{3} \subset \Delta_{k}$ for $k \geqq K_{1}$. Then for all $Z$ in $\Delta^{2}$, we have $\left|Z-a_{i k}\right|$ and $\left|Z-b_{j k}\right|>r$. It follows that

$$
\left|\log T_{k}\left(Z_{2}\right)-\log T_{k}\left(Z_{1}\right)\right| \leqq 2\left|Z_{2}-Z_{1}\right| / r,
$$

for all pairs $Z_{1}, Z_{2}$ of points of $\Delta^{2}$. Let $D^{1}=f^{-1}\left(\Delta^{1}\right)$. There is an integer $K_{2} \geqq K_{1}$ such that for $k \geqq K_{2}, D^{1} \subset D_{k}$ and $\left|f_{k}(z)-f(z)\right|<r$ for all $z$ in $D^{1}$. Since $f(z) \in \Delta^{1}$, $f_{k}(z) \in \Delta^{2}$. Now, using (1) above and the fact that $\log T_{k}\left[f_{k}(z)\right]=u_{k}(z) / \tau_{k}$, we have, for $Z$ in $\Delta^{1}, z=f^{-1}(Z)$ in $D^{1}$, and $k, q \geqq K_{2}$,

$\left|\log T_{k}(Z)-\log T_{q}(Z)\right| \leqq\left|\log T_{k}\left[f_{k}(z)\right]-\log T_{k}[f(z)]\right|$

$$
\begin{aligned}
& +\left|\log T_{q}\left[f_{q}(z)\right]-\log T_{q}[f(z)]\right|+\left|u_{k}(z) / \tau_{k}-u_{q}(z) / \tau_{q}\right| \\
\leqq & 2\left|f_{k}(z)-f(z)\right| / r+2\left|f_{q}(z)-f(z)\right| / r \\
& +\left|u_{k}(z) / \tau_{k}-u_{q}(z) / \tau_{q}\right| .
\end{aligned}
$$

Since the sequences $\left\{f_{k}\right\},\left\{u_{k}\right\}$ converge uniformly on $D^{1}$, and $\left\{\tau_{k}\right\}$ converges to $\tau \neq 0$, it follows from (2) that the sequence $\left\{\log T_{k}\right\}$ has the uniform Cauchy property in $\Delta^{\mathbf{1}}$.

It remains to show that as $k \rightarrow \infty, \log T_{k}(Z) \rightarrow \log T(Z)$ pointwise in $\Delta-\infty$. Fix $Z$ in $\Delta-\infty$. The following inequality is valid:

$$
\begin{aligned}
\left|\log T_{k}(Z)-\log T(Z)\right| \leqq & \left|\log \left(A_{k} \mid A\right)\right| \\
& +\left|\sum_{i=1}^{\mu_{k}} M_{i} \log \right| Z-a_{i k}\left|-\sum_{i=1}^{\infty} M_{i} \log \right| Z-a_{i}|| \\
& +\left|\sum_{j=1}^{v_{k}} N_{j} \log \right| Z-b_{j k}\left|-\sum_{j=1}^{\infty} N_{j} \log \right| Z-b_{j}|| \\
& +\left|\sum_{i=1}^{\mu_{k}}\left(M_{i k}-M_{i}\right) \log \right| Z-a_{i k}\left|-\int \log \right| Z-\alpha|d \mu(\alpha)| \\
& +\left|\sum_{j=1}^{v_{k}}\left(N_{j k}-N_{j}\right) \log \right| Z-b_{j k}\left|-\int \log \right| Z-\alpha|d \nu(\alpha)|
\end{aligned}
$$


We now show that as $k \rightarrow \infty$, all five terms of the right side of the inequality (3) tend to zero.

Since by Proposition 2, $A_{k} \rightarrow A$, it follows that $\log \left(A_{k} / A\right) \rightarrow 0$ as $k \rightarrow \infty$.

Select two rational, half-open rectangles $E_{0}$ and $E$ such that $Z$ is interior to $E_{0}$, $\bar{E}_{0} \subset \Delta$, and $E_{0}, \Delta^{\prime},\left\{a_{i k}\right\},\left\{b_{j k}\right\}$ are all contained in $E$. By Lemma 2 , there is an integer $K_{1}$ such that $\bar{E}_{0} \subset \Delta_{k}$ for $k \geqq K_{1}$. For such $k$, no $a_{i k}$ or $b_{j k}$ is in $E_{0}$; thus for $k \geqq K_{1}$, all $a_{i k}$ and $b_{j k}$ are in $E-E_{0}$. Let $r$ and $R$ be positive numbers such that for all $a$ in $E-E_{0}$, we have $r \leqq|Z-a| \leqq R$.

Let $\varepsilon>0$ be given. Let $q$ be a positive integer such that $\sum_{i=q+1}^{\infty} M_{i}<\varepsilon$. Since $\mu_{k} \rightarrow \infty$, and $a_{i k} \rightarrow a_{i}$, there is an integer $K_{2} \geqq K_{1}$ such that for $k \geqq K_{2}$ and $i=1,2$, $\ldots, q$, we have $\mu_{k} \geqq q$ and $\left|a_{i k}-a_{i}\right|<r \varepsilon$. Then for $k \geqq K_{2}$ the second term of the right side of inequality (3) is less than or equal to

$$
\begin{aligned}
\sum_{i=1}^{q} M_{i}|\log | Z-a_{i k}|-\log | Z-a_{i}||+\sum_{i=q+1}^{\mu_{k}} M_{i}|\log | Z-a_{i k}||+\sum_{i=q+1}^{\infty} M_{i}|\log | Z-a_{i}|| \\
\leqq \\
\sum_{i=1}^{q} M_{i}\left|a_{i k}-a_{i}\right| / r+2 \sum_{i=q+1}^{\infty} M_{i}(|\log r|+|\log R|) \\
<\sum_{i=1}^{q} M_{i} \varepsilon+2(|\log r|+|\log R|) \varepsilon \leqq(1+2|\log r|+2|\log R|) \varepsilon
\end{aligned}
$$

Thus as $k \rightarrow \infty$, the second term tends to zero. The proof for the third term is the same.

The fourth term is next. We use some of the structure that was erected to treat the second term.

Since $Z$ is interior to $E_{0}$, since $m\left(E_{0}\right)=\mu\left(E_{0}\right)=0$, and since $\log |Z-\alpha|$ is continuous except at $\alpha=Z$, we have

$$
\int \log |Z-\alpha| d \mu(\alpha)=M(\log |Z-\alpha|)
$$

Let $\varepsilon>0$ be given. Since it is possible to express $E-E_{0}$ as the disjoint union of four or fewer rational, half-open rectangles, we may, and do, subdivide $E-E_{0}$ into finitely many rational, half-open rectangles $r_{1}, r_{2}, \ldots, r_{t}$, all of diameter $<r \varepsilon$. For each $j$, let $\alpha_{j} \in r_{j}$. We neglect $E_{0}$, since $m\left(E_{0}\right)=0$. Then it is not difficult to show that

$$
\left|M(\log |Z-\alpha|)-\sum_{j=1}^{t} m\left(r_{j}\right) \log \right| Z-\alpha_{j}||<\varepsilon .
$$

Since $m_{k} \rightarrow m$, there is an integer $K_{3} \geqq K_{2}$ such that for $j=1,2, \ldots, t$,

$$
\left|m_{k}\left(r_{j}\right)-m\left(r_{j}\right)\right|<\varepsilon / t .
$$


Then

$$
\begin{aligned}
\left|\sum_{j=1}^{t} m\left(r_{j}\right) \log \right| Z-\alpha_{j}\left|-\sum_{j=1}^{t} m_{k}\left(r_{j}\right) \log \right| Z-\alpha_{j}|| \\
\leqq \sum_{j=1}^{t}\left|m\left(r_{j}\right)-m_{k}\left(r_{j}\right)\right||\log | Z-\alpha_{j}|| \\
\leqq \sum_{j=1}^{t}(|\log r|+|\log R|) \varepsilon / t=(|\log r|+|\log R|) \varepsilon .
\end{aligned}
$$

Now fix $j$ between 1 and $t$. For $k \geqq K_{3}$, the following inequality is valid, where the sum is over all $i$ such that $a_{i k} \in r_{j}$ :

$$
\begin{aligned}
\left|\sum_{i}\left(M_{i k}-M_{i}\right) \log \right| Z-a_{i k} \mid- & m_{k}\left(r_{j}\right) \log \left|Z-\alpha_{j}\right| \mid \\
& \leqq \sum_{i}\left|M_{i k}-M_{i}\right||\log | Z-a_{i k}|-\log | Z-\alpha_{j}|| \\
& \leqq \sum_{i}\left(M_{i k}+M_{i}\right) \frac{\left|a_{i k}-\alpha_{j}\right|}{r} \leqq \sum_{i}\left(M_{i k}+M_{i}\right) \varepsilon .
\end{aligned}
$$

Since for fixed $k \geqq K_{3}$, each $a_{i k}$ is in exactly one rectangle $r_{j}$, it follows that

(7) $\left|\sum_{i=1}^{\mu_{k}}\left(M_{i k}-M_{i}\right) \log \right| Z-a_{i k}\left|-\sum_{j=1}^{t} m_{k}\left(r_{j}\right) \log \right| Z-\alpha_{j}|| \leqq \sum_{i=1}^{\mu_{k}}\left(M_{i k}+M_{i}\right) \varepsilon \leqq 2 \varepsilon$.

Then, as a consequence of (4), (5), (6) and (7), we obtain for $k \geqq K_{3}$,

$$
\left|\sum_{i=1}^{\mu_{k}}\left(M_{i k}-M_{i}\right) \log \right| Z-a_{i k}\left|-\int \log \right| Z-\alpha|d \mu(\alpha)| \leqq(3+|\log r|+|\log R|) \varepsilon .
$$

Therefore, the fourth term tends to zero as $k \rightarrow \infty$. The proof for the fifth term is the same.

This completes the proof of Proposition 16.

Proposition 17. $u(z)=\tau \log T[f(z)]$ for all $z$ in $D$.

Proof. Choose $z$ in $D-\infty$; then $f(z)$ is in $\Delta-\infty$. Let $\varepsilon>0$ be given. Since $f_{k}(z) \rightarrow f(z)$, there is an integer $K_{0}$ such that $f_{k}(z)$ is defined and is in $\Delta-\infty$ for $k \geqq K_{0}$. For $k \geqq K_{0}$, the points $f_{k}(z)$, with the limit $f(z)$, form a compact subset of $\Delta-\infty . \lim _{k \rightarrow \infty} \log T_{k}(Z)=\log T(Z)$ uniformly on this set. There is an integer $K_{1} \geqq K_{0}$ such that for $k \geqq K_{1}$,

$$
\left|\log T_{k}\left[f_{k}(z)\right]-\log T\left[f_{k}(z)\right]\right|<\varepsilon / 2 .
$$

The function $\log T(Z)$ is continuous at $Z=f(z)$. Since $f_{k}(z) \rightarrow f(z)$, there is an integer $K_{2} \geqq K_{1}$ such that for $k \geqq K_{2}$,

$$
\left|\log T\left[f_{k}(z)\right]-\log T[f(z)]\right|<\varepsilon / 2 .
$$


Therefore, if $k \geqq K_{2}$,

$$
\left|\log T_{k}\left[f_{k}(z)\right]-\log T[f(z)]\right|<\varepsilon
$$

Then

$$
u(z) / \tau=\lim _{k \rightarrow \infty} u_{k}(z) / \tau_{k}=\lim _{k \rightarrow \infty} \log T_{k}\left[f_{k}(z)\right]=\log T[f(z)]
$$

thus $u(z)=\tau \log T[f(z)]$ for $z$ in $D-\infty$.

Since $\tau \log T[f(z)] \rightarrow \tau \log A$ as $z \rightarrow \infty$, we define $\tau \log T[f(\infty)]=\tau \log A$. Then letting $z \rightarrow \infty$, we obtain $u(\infty)=\tau \log A$, which coincides with the value for $u(\infty)$ obtained in the proof of Proposition 2. Thus $u(z)=\tau \log T[f(z)]$ for $z$ in $D$.

Proposition 18. $\Delta \subseteq\left\{Z \mid 1<T(Z)<e^{1 / \tau}\right\}$.

Proof. If $Z$ is in $\Delta$, then $z=f^{-1}(Z)$ is in $D$. By Proposition $17, u(z)=\tau \log T(Z)$. Since $0<u(z)<1,0<\tau \log T(Z)<1$; thus $1<T(Z)<e^{1 / \tau}$.

Let $\mu^{*}$ be the measure defined on all Borel subsets $S$ of the extended $Z$-plane by the equation

$$
\mu^{*}(S)=\mu(S)+\sum M_{i},
$$

where the sum is over all $i$ for which $a_{i} \in S$. Then $\mu^{*}(\Delta)=0$. If $S$ is that part of $\Delta^{\prime}$ interior to $\Gamma$, then it is easy to show that $\mu^{*}(S)=1, \mu^{*}\left(S^{\prime}\right)=0$.

Similarly, we define a measure $\nu^{*}$, using the measure $\nu$ and the exponents $N_{j}$. In this case, if $S$ is the part of $\Delta^{\prime}$ exterior to $\Gamma$, then $\nu^{*}(S)=1, \nu^{*}\left(S^{\prime}\right)=0$.

Then we have

$$
\log T(Z)=\log A+\int \log |Z-\alpha| d \mu^{*}(\alpha)-\int \log |Z-\alpha| d \nu^{*}(\alpha) .
$$

It can be shown that $\log T(Z)$ is harmonic in $\Delta-\infty$, subharmonic interior to $\Gamma$, and superharmonic exterior to $\Gamma[1, \mathrm{pp} .135,248]$.

Proposition 19. If $0<\tau \log T(Z)<1$, then $Z$ is in $\Delta$. This, with Proposition 18, implies that $\Delta=\left\{Z \mid 1<T(Z)<e^{1 / \tau}\right\}$.

Proof. Let $Q$ be any component of $\Delta^{\prime}$ that is interior to $\Gamma=f(\gamma)$. Let $q$ be the component of $D^{\prime}$ that corresponds to $Q$. Let $k$ be any positive integer. Exactly one Jordan curve $B_{i k}$ of the locus $u(z)=\varepsilon_{k}$ encloses $q$. Then $\beta=f\left(B_{i k}\right)$ is a Jordan curve in $\Delta$ that encloses $Q$. By Proposition 17, $\tau \log T(Z)=\varepsilon_{k}$ on $\beta$. Since $\tau \log T(Z)$ is subharmonic on the closure of the region interior to $\beta, \tau \log T(Z) \leqq \varepsilon_{k}$ interior to $\beta$. In particular, $\tau \log T(Z) \leqq \varepsilon_{k}$ on $Q$. Since $\varepsilon_{k} \rightarrow 0$ as $k \rightarrow \infty$, we may conclude that $\tau \log T(Z) \leqq 0$ on $Q$. Thus $\tau \log T(Z) \leqq 0$ for $Z$ in $\Delta^{\prime}$ and interior to $\Gamma$.

By similar reasoning, $\tau \log T(Z) \geqq 1$ for $Z$ in $\Delta^{\prime}$ and exterior to $\Gamma$. Consequently, if $0<\tau \log T(Z)<1$, then $Z$ is in $\Delta$.

Define $B^{*}$ as that part of the boundary of $\Delta$ interior to $\Gamma$; define $C^{*}$ as the part exterior to $\Gamma$. 
Proposition 20. $T(Z)$ is continuous in $\bar{\Delta}$, with the value 1 on $B^{*}$ and $e^{1 / \tau}$ on $C^{*}$.

Proof. Let $Z_{0} \in B^{*}$. As in the proof of Proposition 19, $\tau \log T\left(Z_{0}\right) \leqq 0$. Let $Z \in \Delta$; then $\tau \log T(Z)>0$. Since $\tau \log T(Z)$ is upper semicontinuous at $Z_{0}$,

$$
\begin{aligned}
0 & \leqq \liminf _{Z \rightarrow Z_{0}} \tau \log T(Z) \leqq \limsup _{Z \rightarrow Z_{0}} \tau \log T(Z) \\
& \leqq \tau \log T\left(Z_{0}\right) \leqq 0 .
\end{aligned}
$$

It follows that $\lim _{Z \rightarrow Z_{0}} T(Z)=T\left(Z_{0}\right)=1$. Thus $T(Z)$ is continuous in $\Delta \cup B^{*}$ with the value 1 on $B^{*}$.

By similar reasoning, $T(Z)$ is continuous on $\Delta \cup C^{*}$ with the value $e^{1 / \tau}$ on $C^{*}$.

Clearly, $T(Z)$ is continuous on $\bar{\Delta}$.

An immediate consequence of Proposition 20 is that if the exponent $M_{i}>0$, the corresponding point $a_{i}$ is in the exterior of $\Delta$, and thus is an isolated point of $\left\{a_{i}\right\}$. A similar statement holds for $b_{j}$ with $N_{j}>0$.

Proposition 21. For each $Z \neq \infty$, the product $\prod_{i=1}^{\infty}\left|Z-a_{i}\right|^{M_{i}}$ converges absolutely. Also, $\exp \int \log |Z-\alpha| d \mu(\alpha)>0$. Therefore, the zeros of the numerator of $T(Z)$ are exactly those points $a_{i}$ with $M_{i}>0$.

Analogous statements hold for the factors of the denominator of $T(Z)$.

Proof. If $Z$ is not a limit point of $\left\{a_{i}\right\}$, then it is easy to show as in Proposition 6 that the product converges absolutely. Also, the product is zero if and only if $Z$ coincides with some $a_{i}$ with $M_{i}>0$.

Now let $Z$ be a limit point of $\left\{a_{i}\right\}$; thus $Z \in B^{*}$, and $T(Z)=1$. Then we have

$$
\prod_{i=1}^{\infty}\left|Z-a_{i}\right|^{M_{i}} \exp \int \log |Z-\alpha| d \mu(\alpha)>0 .
$$

Since both factors here are nonnegative real numbers, both are positive. Then by Proposition 6 , the product converges absolutely. We have also shown that if $Z \in B^{*}$, then $\exp \int \log |Z-\alpha| d \mu(\alpha)>0$.

Suppose now that $Z \notin B^{*}$. The $\mu$-measures of $\Delta$, of the exterior of $\Delta$, and of that part of the $Z$-plane exterior to the Jordan curve $\Gamma$ are all zero; thus $\mu\left(B^{*^{\prime}}\right)=0$. Since $B^{*}$ is closed, there is a positive distance between $Z$ and $B^{*}$; thus

$$
\exp \int \log |Z-\alpha| d \mu(\alpha)=\exp \int_{B^{*}} \log |Z-\alpha| d \mu(\alpha)>0 .
$$

For fixed $Z, \log |Z-\alpha|$ is $\mu$-integrable.

Proposition 22. $\log T(Z)$ is continuous on each component of $\Delta^{\prime}$, except perhaps for a negative pole at a point $a_{i}$ with $M_{i}>0$, or a positive pole at $b_{j}$ with $N_{j}>0$.

Proof. Let $Q$ be a component of $\Delta^{\prime}$ interior to $\Gamma$. Let $\left\{\beta_{k}\right\}$ be a sequence of analytic Jordan curves in $\Delta$, interior to $\Gamma$, and which contracts to $Q$. For each $k$, let $S_{k}$ be that part of $\Delta^{\prime}$ exterior to $\beta_{k}$. 
There is a positive number $R$ such that for $Z$ in $Q$ and $\alpha$ in $\Delta^{\prime},|Z-\alpha| \leqq R$; thus $|(Z-\alpha) / R| \leqq 1$ and $\log |(Z-\alpha) / R| \leqq 0$. Let

$$
T^{k}(Z)=A R \exp \left[\int_{Q \cup S_{k}} \log \left|\frac{Z-\alpha}{R}\right| d \mu^{*}(\alpha)-\int \log |Z-\alpha| d \nu^{*}(\alpha)\right],
$$

where $\mu^{*}$ and $\nu^{*}$ are the measures defined just before Proposition 19. Then

$$
\log T(Z)-\log T^{k}(Z)=\int_{\Delta^{\prime}-\left(Q \cup S_{k}\right)} \log \left|\frac{Z-\alpha}{R}\right| d \mu^{*}(\alpha) \leqq 0 .
$$

Using Proposition 21, it is not difficult to show that for $Z \in Q, \log T^{k}(Z) \downarrow \log T(Z)$ as $k \rightarrow \infty$.

If no point of $\left\{a_{i}\right\}$ is on $Q$, define $H(Z)=0$ on $Q$. If a point $a_{t}$ is on $Q$, define $H(Z)=M_{t} \log \left|Z-a_{t}\right|+h(Z)$, where $h$ is harmonic on each component of the interior of $Q$, is continuous on $Q$, and takes values on the boundary of $Q$ such that $H=0$ there. Then $\log T^{k}(Z)-H(Z)$ is harmonic in the interior of $Q$ and continuous on $Q$ (except perhaps for a removable singularity at $Z=a_{t}$ ), and is $\geqq 0$ on the boundary of $Q$. Thus $H(Z) \leqq \log T^{k}(Z)$ for $Z$ on $Q$. Letting $k \rightarrow \infty$, obtain $H(Z) \leqq \log T(Z) \leqq 0$. Since $\log T(Z)=0$ on the boundary of $Q$, it follows that $\log T(Z)$ is continuous on $Q$, except perhaps at $a_{t}$. In fact, $\log T(Z)=H(Z)$ on $Q$.

The same reasoning shows that $\log T(Z)$ is continuous on each component of $\Delta^{\prime}$ exterior to $\Gamma$, except for a positive pole at a point $b_{j}$ with $N_{j}>0$. Proposition 22 is established.

Since by Proposition 21, the products in $T(Z)$ converge, and the integrals are finite, any linear transformations of the $Z$-plane can be made, leaving the essential form of $T(Z)$ unchanged. Suppose that $f$ maps $D$ onto the region $\Delta$ defined by $1<T(Z)<e^{1 / \tau}$. Let $g(Z)=(a Z+b) /(c Z+d), a d-b c \neq 0$. Then the function $g f$ maps $D$ onto the region $\Delta^{*}$ defined by $1<T^{*}(Z)<e^{1 / \tau}$, where $T^{*}(Z)=T\left[g^{-1}(Z)\right]$. In the case where $g\left(a_{i}\right)$ and $g\left(b_{j}\right) \neq \infty$ we obtain

$$
T^{*}(Z)=T(-d / c) \frac{\prod_{i}\left|Z-g\left(a_{i}\right)\right|^{M_{i}} \exp \int \log |Z-\beta| d \mu_{*}(\beta)}{\prod_{j}\left|Z-g\left(b_{j}\right)\right|^{N_{j}} \exp \int \log |Z-\beta| d \nu_{*}(\beta)},
$$

where $\mu_{*}$ and $\nu_{*}$ are Borel measures such that for every Borel set $S$ in the extended $Z$-plane,

$$
\mu_{*}(S)=\mu\left[g^{-1}(S)\right], \quad \nu_{*}(S)=\nu\left[g^{-1}(S)\right] .
$$

The homeomorphic image of a Borel set is a Borel set [6, pp. 237, 238].

This completes the proof of Theorem 2.

One shortcoming of the above proof is that we have not shown that the boundary of $\Delta$ consists exactly of the two $\operatorname{loci} \tau \log T(Z)=0$ and $\tau \log T(Z)=1$. In view of Proposition 22, in order to establish this, it would be sufficient to show that $T(Z)$ is not constant on any open disk. Whether or not this is the case is not now known to the author. Of some interest is the fact that each of the above loci is a closed set. The proof resembles that of Proposition 20. 
Here are two examples that illustrate the extremes possible in the form of $T(Z)$ in Theorem 2.

(1) If the region $D$ is of countably infinite connectivity, then the measures $\mu$ and $\nu$ are zero; thus the integrals in $T(Z)$ are zero. Furthermore, it can be shown that in this case there are infinitely many isolated boundary components of $D$; hence there are infinitely many nonzero exponents $M_{i}$ and $N_{j}$. Therefore $T(Z)$ is the product of the constant $A$ and a quotient of infinite products.

(2) Let $D$ be the complement (relative to the extended plane) of the ordinary Cantor set on the real interval $[0,1]$. Surprisingly, $D$ is a Dirichlet region. A detailed discussion of this, with proofs, can be found in [1, p. 252]. Then, as asserted after the proof of Proposition 3 (of Theorem 2), all the exponents $M_{i}$ and $N_{j}$ are zero. Thus, in this second case, it is the products that do not occur in $T(Z)$.

As in [11] and [12], Theorem 2 extends to the limiting case where the components of $B$ reduce to points. The proof is based on the next theorem, due also to $J$. L Walsh [11, Theorem 3].

THEOREM 3 (WALSH). Let $D$ be a region of the extended z-plane whose boundary consists of mutually disjoint Jordan curves $C_{1}, C_{2}, \ldots, C_{v}$. Let $\alpha_{1}, \alpha_{2}, \ldots, \alpha_{\mu}$ be distinct points of $D$; and let $M_{1}, M_{2}, \ldots, M_{\mu}$ be positive numbers such that $\sum M_{i}=1$. There exists a conformal map $f$ of $D$ onto a region $\Delta$ of the extended $Z$-plane, oneto-one and continuous in the closed regions, where $\Delta$ is defined by

$$
T(Z)<1, \quad T(Z)=A \frac{\prod_{i=1}^{\mu}\left|Z-a_{i}\right|^{M_{i}}}{\prod_{j=1}^{v}\left|Z-b_{j}\right|^{N_{j}}}
$$

with $A, N_{j}>0, \sum N_{j}=1$, and $a_{i}=f\left(\alpha_{i}\right)$. The locus $T(Z)=1$ consists of $\nu$ analytic Jordan curves, which are respective images of the $C_{j}$, and which separate $\Delta$ from the $b_{j}$.

In Theorem 4 below, we replace $\left\{\alpha_{i}\right\}$ by a compact subset $B$ of $D$, and the exponents $\left\{M_{i}\right\}$ by a Borel measure $\mu_{*}$ such that $\mu_{*}(B)=1, \mu_{*}\left(B^{\prime}\right)=0$.

TheOREm 4. Let $D$ be a Dirichlet region in the extended z-plane. Let $B$ be a nonempty compact subset of $D$; let $C$ be the boundary of $D$. Let $\mu_{*}$ be a Borel measure in the extended z-plane such that $\mu_{*}(B)=1, \mu_{*}\left(B^{\prime}\right)=0$. There exists a conformal map $f$ of $D$ onto a region $\Delta$ of the extended $Z$-plane, where $\Delta$ is defined by

$$
T(Z)<1, \quad T(Z)=A \frac{\exp \int \log |Z-\alpha| d \mu(\alpha)}{\prod_{j}\left|Z-b_{j}\right|^{N_{j}} \exp \int \log |Z-\alpha| d \nu(\alpha)},
$$

with $A, N_{j}>0$; and $\sum_{j} N_{j} \leqq 1$. The measure $\mu$ is a Borel measure such that $\mu(S)$ $=\mu_{*}\left[f^{-1}(S)\right]$ for every Borel set $S$ in the extended Z-plane. The measure $v$ is a regular Borel measure such that $\nu(\Delta)=0, \nu(Z$-plane $)=1-\sum_{j} N_{j}$, the v-measure of each component of $\Delta^{\prime}$ is zero, the $\nu$-measure of the exterior of $\Delta$ is zero. The function $T(Z)$ is continuous on $\bar{\Delta}-f(B)$, with the value 1 on the boundary of $\Delta ; T(Z)$ is 
continuous on each component of $\Delta^{\prime}$ (except at the $b_{j}$ ). The $b_{j}$ are distinct, isolated points and are separated from each other and from $\Delta$ by the locus $T(Z)=1$.

Proof. The proof follows closely that of Theorem 2. Most of the propositions are the same, and have the same, or similar proofs. As in the proof of Theorem 2, we shall assume that the boundary $C$ consists of infinitely many components. Modifications of the proof can be made if otherwise. We assume that 0,1 are in $D$ and that $\infty$ is in $D-B$, since this may be effected by a preliminary linear transformation of the extended $z$-plane. One consequence of this is that $B \cup C$ is bounded.

Let $v$ be the unique function harmonic in $D-\infty$, continuous in $\bar{D}-\infty$, with a negative pole at $\infty$, and such that if we set

$$
u(z)=v(z)+\int \log |z-\alpha| d \mu_{*}(\alpha)
$$

then $u=0$ on $C$, and has a finite limit $u(\infty)$ at $\infty$. The function $u$ is harmonic in $(D-\infty)-B$ and continuous in $\bar{D}-B$. Furthermore, the integral in $u$ is subharmonic [1, pp. 135, 248]; thus $u$ is upper semicontinuous in $\bar{D}$ and subharmonic in $D-\infty$.

Proposition 1. $u(z)<0$ for $z$ in $D$.

Proof. Since $u$ is subharmonic in $D-\infty, u$ is either constant or fails to attain a maximum in $D-\infty$.

Suppose $u$ is constant in $D-\infty ; u=K$. Then we have

$$
\int \log |z-\alpha| d \mu_{*}(\alpha)=K-v(z)
$$

Since $K-v$ is harmonic in $D-\infty$ and the integral is harmonic in $B^{\prime}-\infty$, the integral is harmonic in the $z$-plane with a positive pole at $\infty$. However, no such function exists; thus $u$ is not constant in $D-\infty$.

The alternative is that $u$ have no maximum in $D-\infty$. Since $u$ is harmonic and bounded in a neighborhood of $\infty, u$ has no maximum in $D$. Since $u$ is upper semicontinuous in $\bar{D}, u$ does attain a maximum in $\bar{D}$. Then since $u=0$ on $C$, it follows that $u \leqq 0$ in $\bar{D}$, and $u<0$ in $D$. Proposition 1 is established.

Now we define the regions $D_{k}$. An application of Lemma 1 to each component of $C$, followed by a standard compactness argument, shows that there exists a finite collection $\left\{\gamma_{1}, \gamma_{2}, \ldots, \gamma_{q}\right\}$ of Jordan curves in $D-\infty$ such that every point of $C$ is interior to at least one $\gamma_{i}$ and no point of $B \cup\{0,1\}$ is either on or interior to any $\gamma_{i}$. Let $\gamma$ be the union of all the $\gamma_{i}$. Then $\gamma$ is a compact subset of $D-B$.

Let $\left\{\varepsilon_{k}\right\}$ be a strictly increasing sequence of negative numbers with limit 0 and such that $\varepsilon_{1}$ is larger than the maximum value of $u$ on $\gamma$. Then it can be shown that for each $k$, every point of the locus $u=\varepsilon_{k}$ is interior to at least one of the curves $\gamma_{i}$. For each $i, u$ is harmonic and not constant in the subregion of $D$ interior to $\gamma_{i}$. Hence there are at most countably many critical points of $u$ interior to the $\gamma_{i}$. 
Therefore we may suppose further that for every integer $k$, there is no critical point of $u$ on the locus $u(z)=\varepsilon_{k}$. Then, as in the proof of Theorem 2, for each $k$, the locus $u(z)=\varepsilon_{k}$ consists of finitely many, mutually exterior, analytic Jordan curves in $D-B$ and separating $C$ from $B$. Each curve of the locus is interior to at least one of the curves $\gamma_{i}$.

Let $D_{k}=\left\{z \mid u(z)<\varepsilon_{k}\right\}$. Each $D_{k}$ is a finitely connected subregion of $D$ containing the points $0,1, \infty$, as well as the set $B$. The boundary of $D_{k}$ consists of the locus $u(z)=\varepsilon_{k}$. Since $\varepsilon_{k} \uparrow 0$ as $k \rightarrow \infty, D_{1} \subseteq D_{k} \subset D_{k+1} \subset \ldots \subset D$, and $\lim _{k \rightarrow \infty} D_{k}=D$.

As in the proof of Theorem 2, the nonnegative number $(-1) \int_{E} \partial u / \partial n d s$ is defined for each component $E$ of the boundary $C$. Then for each $k$, the Jordan curves of the boundary of $D_{k}$ are carefully labeled as $C_{j k}\left(j=1,2, \ldots, \nu_{k}\right)$. Most important is that for each $j=1,2, \ldots, v_{k}, C_{j k+1}$ be interior to $C_{j k}$, and separate $C_{j k}$ from a boundary component $E$ interior to $C_{j k}$ for which the number

$$
(-1) \int_{E} \frac{\partial u}{\partial n} d s
$$

is maximal. Since we have assumed that $D$ is infinitely connected, $\nu_{k} \rightarrow \infty$ as $k \rightarrow \infty$. Finally, some but not necessarily all of the components of $C$ are labeled $C_{1}, C_{2}, C_{3}, \ldots$ For fixed $j$, the sequence $C_{j k}$ contracts to $C_{j}$ as $k \rightarrow \infty$.

The next step is to define a suitable sequence of finite subsets of $B$. Each of these sets will correspond to the set $\left\{\alpha_{i}\right\}$ in Theorem 3. For $t=1,2,3, \ldots$, let $P_{t}$ be a subdivision of $B$ into finitely many, mutually disjoint, nonempty Borel subsets $B_{i t}$ $\left(i=1,2, \ldots, \mu_{t}\right)$ such that norm $P_{t} \rightarrow 0$ as $t \rightarrow \infty$. For $i=1,2, \ldots, \mu_{t}$, let $\alpha_{i t}$ be a point of $B_{i t}$. Define $M_{i t}=\mu_{*}\left(B_{i t}\right)$. For each $t, \sum_{i=1}^{\mu_{t}} M_{i t}=1$.

We now choose a subsequence $\left\{P_{t_{k}}\right\}$ of $\left\{P_{t}\right\}$ as follows. Fix the integer $k$; and suppose that integers $t_{i}(1 \leqq i<k)$ have been selected according to the scheme below. Let $Q$ be the set that consists of every point of the $z$-plane that is either on or interior to at least one of the curves $\gamma_{i}(i=1,2, \ldots, q)$. Then $Q$ is compact, bounded, and disjoint from $B$. Since the distance between $B$ and $Q$ is positive and since norm $P_{t} \rightarrow 0$ as $t \rightarrow \infty$, it can be easily shown that

$$
\lim _{t \rightarrow \infty} \sum_{i=1}^{\mu_{t}} M_{i t} \log \left|z-\alpha_{i t}\right|=\int \log |z-\alpha| d \mu_{*}(\alpha)
$$

uniformly for $z$ in $Q$. Then for $t=1,2,3, \ldots$, let

$$
w_{t}(z)=h_{t}(z)+\sum_{i=1}^{\mu_{t}} M_{i t} \log \left|z-\alpha_{i t}\right|,
$$

where $h_{t}$ is harmonic in $D_{k}-\infty$, is continuous in $\bar{D}_{k}-\infty$ with values on the boundary of $D_{k}$ such that $w_{t}=0$ there, and has a negative pole at $\infty$ such that $w_{t}$ has a finite limit at $\infty$. Since $w_{t}=0$ and $u=\varepsilon_{k}$ on the boundary of $D_{k}$,

$$
h_{t}(z)-v(z)=-\varepsilon_{k}+\int \log |z-\alpha| d \dot{\mu}_{*}(\alpha)-\sum_{i=1}^{\mu_{t}} M_{i t} \log \left|z-\alpha_{i t}\right| .
$$


Since $Q$ contains the boundary of $D_{k}, \lim _{t \rightarrow \infty}\left[h_{t}(z)-v(z)\right]=-\varepsilon_{k}$ uniformly on the boundary of $D_{k}$. Since $h_{t}-v$ is harmonic in $D_{k}-\infty$ and has a finite limit at $\infty$, the convergence to $-\varepsilon_{k}$ is uniform in $\bar{D}_{k}-\infty$. Since

$$
w_{t}(z)=u(z)+h_{t}(z)-v(z)+\sum_{i=1}^{\mu_{t}} M_{i t} \log \left|z-\alpha_{i t}\right|-\int \log |z-\alpha| d \mu_{*}(\alpha),
$$

it follows that $\lim _{t \rightarrow \infty} w_{t}(z)=u(z)-\varepsilon_{k}$ uniformly in $D_{k} \cap Q$. For each $j=1,2, \ldots, v_{k}$, let $\beta_{j}$ be an analytic Jordan curve in the interior of $Q$ which encloses the boundary curve $C_{j k}$ and separates $C_{j k}$ from all other boundary components of $D_{k}$. Since $\lim _{t \rightarrow \infty} w_{t}(z)=u(z)-\varepsilon_{k}$ uniformly in a neighborhood of $\beta_{j}$,

$$
\lim _{t \rightarrow \infty} \int_{\beta_{j}} \frac{\partial w_{t}}{\partial n} d s=\int_{\beta_{j}} \frac{\partial u}{\partial n} d s=\int_{C_{j k}} \frac{\partial u}{\partial n} d s
$$

Let $t_{k}$ be the first integer $>t_{k-1}(>0$ if $k=1)$ for which

$$
\left|\int_{\beta_{j}} \frac{\partial w_{t_{k}}}{\partial n} d s-\int_{C_{j k}} \frac{\partial u}{\partial n} d s\right|<1 / k
$$

for $j=1,2, \ldots, \nu_{k}$. These inequalities are necessary in the proof of Proposition 3.

Let $u_{k}=w_{t_{k}}$; let $v_{k}=h_{t_{k}}$. Also, at this point, we abandon the sequence $\left\{P_{t}\right\}$ and reindex the subsequence $\left\{P_{t_{k}}\right\}$, as well as $\left\{B_{i t_{k}}\right\},\left\{\mu_{t_{k}}\right\},\left\{\alpha_{i t_{k}}\right\}$ and $\left\{M_{i t_{k}}\right\}$, obtaining $\left\{\boldsymbol{P}_{k}\right\},\left\{\boldsymbol{B}_{i k}\right\},\left\{\mu_{k}\right\},\left\{\alpha_{i k}\right\}$, and $\left\{\boldsymbol{M}_{i k}\right\}$ respectively. Of course, the sequence of finite subsets of $B$ is $\left\{\alpha_{i k}\right\}, k=1,2,3, \ldots$

Methods similar to those used above show that $\lim _{k \rightarrow \infty} v_{k}=v$ uniformly on each compact subset of $D-\infty$, and $\lim _{k \rightarrow \infty} u_{k}=u$ uniformly on each compact subset of $D-B$. In particular, $\lim _{k \rightarrow \infty} u_{k}(\infty)=u(\infty)$.

For each $k=1,2,3, \ldots, D_{k}$ is bounded by finitely many, mutually disjoint Jordan curves $C_{j k}\left(1 \leqq j \leqq \nu_{k}\right)$. The set $\left\{\alpha_{i k} \mid 1 \leqq i \leqq \mu_{k}\right\}$ is finite. Since $M_{i k} \geqq 0$ and $\sum_{i=1}^{\mu_{k}} M_{i k}=1$, not all $M_{i k}$ equal zero. Thus by Theorem 3 for each region $D_{k}$, there are functions $f_{k}$ and $T_{k}$ such that $f_{k}$ maps $D_{k}$ conformally (except at $\infty$ ) onto a region $\Delta_{k}$ of the extended $Z$-plane, where $\Delta_{k}$ is defined by

$$
T_{k}(Z)<1, \quad T_{k}(Z)=A_{k} \frac{\prod_{i=1}^{\mu_{k}}\left|Z-a_{i k}\right|^{M_{i k}}}{\prod_{j=1}^{\nu_{k}}\left|Z-b_{j k}\right|^{N_{j k}}}
$$

with $A_{k}, N_{j k}>0, \sum N_{j k}=1$, and $a_{i k}=f_{k}\left(\alpha_{i k}\right)$. As before, we assume that $f_{k}(0)=0$, $f_{k}(1)=1$, and $f_{k}(\infty)=\infty$ since this may be obtained by a linear transformation of the $Z$-plane. The map $f_{k}$ is one-to-one and continuous from $\bar{D}_{k}$ onto $\bar{\Delta}_{k}$. The locus $T_{k}(Z)=1$ consists of $\nu_{k}$ mutually exterior, analytic Jordan curves $C_{j k}^{*}$, respective images of the $C_{j k}$, which separate $\Delta_{k}$ from the points $b_{j k}$.

Fix the positive integers $i$ and $k$, with $1 \leqq i \leqq \mu_{k}$. Then for $z$ in a neighborhood of $\alpha_{i k}$,

$$
\log T_{k}\left[f_{k}(z)\right]-u_{k}(z)=M_{i k} \log \left|\frac{f_{k}(z)-f_{k}\left(\alpha_{i k}\right)}{z-\alpha_{i k}}\right|+h_{i k}(z)
$$


where $h_{i k}$ is harmonic in the neighborhood. Then

$$
\lim _{z \rightarrow \alpha_{t k}}\left[\log T_{k}\left[f_{k}(z)\right]-u_{k}(z)\right]=M_{i k} \log \left|f_{k}^{\prime}\left(\alpha_{i k}\right)\right|+h_{i k}\left(\alpha_{i k}\right) ;
$$

hence there is a removable singularity at each $\alpha_{i k}$. As $z \rightarrow \infty$,

$$
\log T_{k}\left[f_{k}(z)\right]-u_{k}(z) \rightarrow \log A_{k}-u_{k}(\infty) ;
$$

thus $\infty$ is also a removable singularity. Finally, both $\log T_{k}\left[f_{k}(z)\right]$ and $u_{k}(z)$ equal 0 on the boundary of $D_{k}$. Therefore $u_{k}(z)=\log T_{k}\left[f_{k}(z)\right]$ for $z$ in $\bar{D}_{k}$.

Proposition 2. $\lim _{k \rightarrow \infty} A_{k}=A$ exists $; 0<A<1$.

Proof. Since $u_{k}(\infty)=\log T_{k}(\infty)=\log A_{k}, A_{k}=\exp u_{k}(\infty)$. Thus $\lim _{k \rightarrow \infty} A_{k}=$ $\exp u(\infty)=A$ exists. Since $-\infty<u(\infty)<0,0<A<1$.

Proposition 3. $\lim _{k \rightarrow \infty} N_{j k}=N_{j}$ exists. $N_{j} \geqq 0 ; \sum_{j} N_{j} \leqq 1$.

Proof. Choose $j$. For $k$ sufficiently large, $j \leqq v_{k}$. Let $\beta$ be an analytic Jordan curve in $D_{k}$, enclosing the boundary component $C_{j k}$ and separating $C_{j k}$ from all other boundary components of $D_{k}$ and from $B$. Then, as in Proposition 3, Theorem 2,

$$
N_{j k}=-\frac{1}{2 \pi} \int_{\beta} \frac{\partial u_{k}}{\partial n} d s
$$

As a consequence of (8),

$$
\left|N_{j k}+\frac{1}{2 \pi} \int_{C_{j k}} \frac{\partial u}{\partial n} d s\right|<\frac{1}{2 \pi k} .
$$

As $k \rightarrow \infty, C_{j k}$ contracts to $C_{j}$. It follows that

$$
\lim _{k \rightarrow \infty} N_{j k}=-\frac{1}{2 \pi} \int_{C_{j}} \frac{\partial u}{\partial n} d s=N_{j}
$$

exists.

The proof that $N_{j} \geqq 0$, and $\sum_{j} N_{j} \leqq 1$ is the same as in Proposition 3, Theorem 2 .

Just as in the proof of Theorem 2 , since $f_{k}(0)=0, f_{k}(1)=1$, and $f_{k}(\infty)=\infty$, the family $\left\{f_{k}\right\}$ is normal in $D-\infty$. There is a subsequence $\left\{k^{\prime}\right\}$ of the positive integers and a function $f$ such that $\lim _{k^{\prime} \rightarrow \infty} f_{k^{\prime}}(z)=f(z)$ uniformly on each compact subset of $D-\infty$. The limit function $f$ is not identically constant and thus is one-to-one and analytic in $D-\infty$. As before, defining $f(\infty)=\infty$, we obtain a univalent map of $D$ onto a region $\Delta$ of the extended $Z$-plane. The map is conformal in $D-\infty$, and continuous in $D$.

For $k=1,2,3, \ldots$, and for each rational half-open rectangle $R$ in the $Z$-plane, let $n_{k}(R)=\sum_{j}\left(N_{j k}-N_{j}\right)$, where the sum is over all $j$ such that $b_{j k} \in R$.

Just as in the proof of Theorem 2, we may restrict ourselves henceforth to a definite subsequence of integers $k$ such that $\lim _{k \rightarrow \infty} n_{k}(R)=n(R)$ exists for each rational half-open rectangle $R, \lim _{k \rightarrow \infty} b_{j k}=b_{j}$ exists for each $j$, and $\lim _{k \rightarrow \infty} f_{k}(z)$ $=f(z)$ uniformly on each compact subset of $D-\infty$. 
We may also extend the set function $n$ to all sets $S$ in the $Z$-plane for which $\lim _{k \rightarrow \infty} n_{k}(S)$ exists by the equation $n(S)=\lim _{k \rightarrow \infty} n_{k}(S)$.

To complete the proof, propositions analogous to those of Theorem 2 can be stated. In general, the proofs are similar. Hence, we omit most of the details. However, we shall state the sixteenth proposition, and give a partial proof.

Exactly as in the proof of Theorem 2, the set function $n$ is used to define a linear functional $N$. Then by Riesz's representation theorem, there is a regular Borel measure $\nu$ such that $N(\phi)=\int \phi d \nu$ for every real-valued function $\phi$ continuous in the complex plane.

If $S$ is a Borel subset of the extended $Z$-plane, then $S \cap \Delta$ is a Borel subset of $\Delta$. Then, since $f$ is a homeomorphism, $f^{-1}(S)$ is a Borel subset of $D$, and thus also of the extended $z$-plane [6, pp. 237, 238]. Let $\mu(S)=\mu_{*}\left[f^{-1}(S)\right]$ for every Borel set $S$ in the extended $Z$-plane. Then $\mu$ is a Borel measure such that $\mu\left(B^{*}\right)=1$, $\mu\left(B^{* \prime}\right)=0$, where $B^{*}=f(B)$.

Proposition 16. Let

$$
T(Z)=A \frac{\exp \int \log |Z-\alpha| d \mu(\alpha)}{\prod_{j=1}^{\infty}\left|Z-b_{j}\right|^{N_{j}} \exp \int \log |Z-\alpha| d \nu(\alpha)} .
$$

Let $\Delta^{1}$ be a compact subset of $(\Delta-\infty)-B^{*}$. Then $\lim _{k \rightarrow \infty} \log T_{k}(Z)=\log T(Z)$ uniformly on $\Delta^{1}$.

Proof. First, it is possible to show that the sequence $\left\{\log T_{k}\right\}$ has the uniform Cauchy property on $\Delta^{1}$. The proof used in Theorem 2 is applicable, although several minor modifications are necessary. We omit the details.

The second part of the proof is to show that as $k \rightarrow \infty, \log T_{k} \rightarrow \log T$ pointwise in $(\Delta-\infty)-B^{*}$. Choose $Z$ in $(\Delta-\infty)-B^{*}$. The following inequality is valid:

$$
\begin{aligned}
\left|\log T_{k}(Z)-\log T(Z)\right| \leqq & \left|\log \left(A_{k} / A\right)\right| \\
& +\left|\sum_{j=1}^{v_{k}} N_{j} \log \right| Z-b_{j k}\left|-\sum_{j=1}^{\infty} N_{j} \log \right| Z-b_{j}|| \\
& +\left|\sum_{j=1}^{v_{k}}\left(N_{j k}-N_{j}\right) \log \right| Z-b_{j k}\left|-\int \log \right| Z-\alpha|d \nu(\alpha)| \\
& +\left|\sum_{i=1}^{\mu_{k}} M_{i k} \log \right| Z-a_{i k}\left|-\int \log \right| Z-\alpha|d \mu(\alpha)| .
\end{aligned}
$$

It remains to show that each of the four terms to the right of this inequality tends to zero as $k \rightarrow \infty$. For the first three terms, the problem is exactly as in Theorem 2 . The last term is treated below.

Let $\varepsilon>0$ be given. Let $2 r$ be the distance from $Z$ to $B^{*}$. For each $k$, let $P_{k}^{*}$ be the subdivision of $B^{*}$ into finitely many, mutually disjoint, Borel subsets $B_{i k}^{*}$ $\left(i=1,2, \ldots, \mu_{k}\right)$, with $B_{i k}^{*}=f\left(B_{i k}\right)$, where the $B_{i k}$ are the subsets of $B$ corresponding to the subdivision $P_{k}$. Since $f$ is uniformly continuous on $B$, and since norm $P_{k} \rightarrow 0$, 
it follows that norm $P_{k}^{*} \rightarrow 0$ as $k \rightarrow \infty$. There is an integer $K_{1}$ such that for $k \geqq K_{1}$, norm $P_{k}^{*}<\varepsilon r / 2$.

Since $f_{k} \rightarrow f$ uniformly on $B$, there is an integer $K_{2} \geqq K_{1}$ such that for $k \geqq K_{2}$, $\left|f_{k}(z)-f(z)\right|<\min \{r, \varepsilon r / 2\}$ for all $z$ in $B$. Since $\alpha_{i k} \in B$, and $f_{k}\left(\alpha_{i k}\right)=a_{i k}$, we have $\left|a_{i k}-f\left(\alpha_{i k}\right)\right|<r$ for $k \geqq K_{2}$. Then since $f\left(\alpha_{i k}\right) \in B^{*}$, it follows that $\left|Z-a_{i k}\right| \geqq r$. We also have $|Z-\alpha| \geqq 2 r$ for all $\alpha$ in $B^{*}$.

Since $\alpha_{i k} \in B_{i k}, f\left(\alpha_{i k}\right) \in B_{i k}^{*}$. For $k \geqq K_{2}$, the diameter of $B_{i k}^{*}$ is $<\varepsilon r / 2$; thus $\left|f\left(\alpha_{i k}\right)-\alpha\right|<\varepsilon r / 2$ for all $\alpha$ in $B_{i k}^{*}$. Then since $\left|a_{i k}-f\left(\alpha_{i k}\right)\right|<\varepsilon r / 2$, we have $\left|a_{i k}-\alpha\right|<\varepsilon r$ for all $\alpha$ in $B_{i k}^{*}$. Therefore, since $M_{i k}=\mu_{*}\left(B_{i k}\right)=\mu\left(B_{i k}^{*}\right)$, we obtain for $k \geqq K_{2}$,

$$
\begin{aligned}
\left|\sum_{i=1}^{\mu_{k}} M_{i k} \log \right| Z-a_{i k}\left|-\int \log \right| Z-\alpha|d \mu(\alpha)| \\
\leqq \sum_{i=1}^{\mu_{k}} \int_{B_{i k}^{*}}|\log | Z-a_{i k}|-\log | Z-\alpha|| d \mu(\alpha) \\
\leqq \sum_{i=1}^{\mu_{k}} \int_{B_{i k}^{*}} \frac{\left|a_{i k}-\alpha\right|}{r} d \mu(\alpha) \leqq \sum_{i=1}^{\mu_{k}} \int_{B_{i k}^{*}} \varepsilon d \mu(\alpha)=\varepsilon .
\end{aligned}
$$

Thus, the fifth term tends to zero as $k \rightarrow \infty$. Proposition 16 is established.

The rest of the proof of Theorem 4 is omitted, since it resembles closely that of Theorem 2.

Certain linear transformations of the $Z$-plane can be made, leaving the essential form of $T(Z)$ unchanged. Such a transformation $g(Z)$ is permissible provided that $g^{-1}(\infty)$ is not a point at which $\int \log |Z-\alpha| d \mu(\alpha)=-\infty$. Such points, if any exist, are in $B^{*}$.

This completes the proof of Theorem 4 .

The next theorem is a converse of Theorem 2 .

THEOREM 5. Let $D$ be a region of the extended z-plane. Let there exist a conformal map $f$ of $D$ onto a region $\Delta$ of the extended Z-plane. Suppose that $\Delta^{\prime}$ is the union of two disjoint, nonempty, closed, bounded sets $B^{*}$ and $C^{*}$. Let $Z \in \Delta$ if and only if $0<H(Z)<1$, where $H$ is a function harmonic in $\Delta$ with finite limit at $\infty$, subharmonic in $\Delta \cup B^{*}$, and superharmonic in $\Delta \cup C^{*}$. Then $D$ is a Dirichlet region.

Proof. Let $\Gamma$ be a Jordan curve in $\Delta$ which encloses $B^{*}$, separating $B^{*}$ from $C^{*}$. Let $\rho$ be the maximum value of $H(Z)$ on $\Gamma ; 0<\rho<1$. Since $H(Z)$ is subharmonic interior to $\Gamma, H(Z) \leqq \rho$ interior to $\Gamma$, and thus on $B^{*}$. Then since for each $Z \in \Delta^{\prime}$, either $H(Z) \leqq 0$ or $H(Z) \geqq 1$, we have $H(Z) \leqq 0$ on $B^{*}$. By similar reasoning, $H(Z)$ $\geqq 1$ on $C^{*}$. Since $H(Z)$ is upper semicontinuous interior to $\Gamma$, lower semicontinuous exterior to $\Gamma$, and $0<H(Z)<1$ in $\Delta$, it follows as in the proof of Proposition 20, Theorem 2, that $H$ is continuous in $\bar{\Delta}$, and takes the value 0 at boundary points of $\Delta$ interior to $\Gamma$, and 1 at boundary points exterior to $\Gamma$.

For $z \in D$, let $h(z)=H[f(z)]$. The boundary of $D$ can be separated into two parts $B$ and $C$ that correspond under the conformal map $f$ to the boundaries of $B^{*}$ and 
$C^{*}$ respectively. As $z$ in $D$ tends to $B, h(z) \rightarrow 0$; as $z$ tends to $C, h(z) \rightarrow 1$. Define $h(z)=0$ on $B,=1$ on $C$. Then $h$ is the harmonic measure of $C$ with respect to the region $D$.

We shall show that there is a barrier at every point $P$ on the boundary of $D$. If the boundary component containing such a point $P$ does not reduce to a single point, then there does exist a barrier at $P[1, \mathrm{p} .141]$. Now suppose that the boundary component consists only of $P \neq \infty$. Let $\left\{\beta_{k}\right\}$ be a sequence of Jordan curves in $D$ which contracts to $P$. For $k=1,2,3, \ldots$, let $g_{k}$ be the unique function bounded and harmonic in $D$ (except perhaps at $\infty$ ) which takes the boundary value 0 at the boundary points of $D$ interior to $\beta_{k}$, and the value 1 at boundary points exterior to $\beta_{k}$. The existence of the $g_{k}$ follows from the existence of the nontrivial harmonic measure $h$ above. We omit the proof of this, which consists mainly of perseverance in the application of Harnack's principle. Let $g(z)=\sum_{k=1}^{\infty} 2^{-k} g_{k}(z)$. Since this series converges uniformly in $\bar{D}, g$ is harmonic in $D$ (except perhaps at $\infty$ ) and continuous in $\bar{D}$. Since $g(P)=0$, and $0<g(z) \leqq 1$ for every other point $z$ in $\bar{D}, g$ is a barrier at $P$. In case $P=\infty$, the sequence $\left\{\beta_{k}\right\}$ should expand to $P$. Otherwise, the proof is the same. It follows that $D$ is a Dirichlet region.

A similar theorem, the converse of Theorem 4, is also valid. We omit the statement and proof.

\section{BIBLIOGRAPHY}

1. L. V. Ahlfors and L. Sario, Riemann surfaces, Princeton Math. Series, no. 26, Princeton Univ. Press, Princeton, N. J., 1960. MR 22 \#5729.

2. C. Carathéodory, Conformal representation, 2nd ed., Cambridge Tracts in Math. and Math. Phys., no. 28, Cambridge Univ. Press, New York, 1952. MR 13, 734.

3. H. S. M. Coxeter, Introduction to geometry, Wiley, New York, 1961. MR 23 \#A1251.

4. H. Grunsky, Über konforme Abbildungen, die gewisse Gebietsfunktionen in elementare Funktionen transformieren. I, Math. Z. 67 (1957), 129-132. MR 19, 538.

5. P. R. Halmos, Measure theory, Van Nostrand, Princeton, N. J., 1950. MR 11, 504.

6. F. Hausdorf, Mengenlehre, de Gruyter, Berlin, 1937; English transl., Chelsea, New York, 1957. MR 19, 111.

7. J. A. Jenkins, On a canonical conformal mapping of J. L. Walsh, Trans. Amer. Math. Soc. 88 (1958), 207-213. MR 19, 538.

8. H. J. Landau, On canonical conformal maps of multiply connected domains, Trans. Amer. Math. Soc. 99 (1961), 1-20. MR 22 \#12212.

9. R. L. Moore, Concerning the separation of point sets by curves, Proc. Nat. Acad. Sci. U.S.A. 11 (1925), 469-476.

10. —, Foundations of point set theory, rev. ed., Amer. Math. Soc. Colloq. Publ., vol. 13, Amer. Math. Soc., Providence, R. I., 1962.

11. J. L. Walsh, On the conformal mapping of multiply connected regions, Trans. Amer. Math. Soc. 82 (1956), 128-146. MR 18, 290.

12. J. L. Walsh and H. J. Landau, On canonical conformal maps of multiply connected regions, Trans. Amer. Math. Soc. 93 (1959), 81-96. MR 28 \#4093.

Portland State University, PORTLAND, OREgon 97207 